\title{
PD-1 is a haploinsufficient suppressor of T cell lymphomagenesis
}

\author{
Tim Wartewig ${ }^{1}$, Zsuzsanna Kurgyis ${ }^{1}$, Selina Keppler ${ }^{1}$, Konstanze Pechloff ${ }^{1,5}$, Erik \\ Hameister $^{1}$, Rupert Öllinger ${ }^{2}$, Roman Maresch ${ }^{2}$, Thorsten Buch ${ }^{3}$, Katja Steiger ${ }^{4}$, Christof \\ Winter ${ }^{1}$, Roland Rad $^{2,5}$, and Jürgen Ruland ${ }^{1,5,6}$ \\ ${ }^{1}$ Institut für Klinische Chemie und Pathobiochemie, Klinikum rechts der Isar, Technische \\ Universität München, 81675 München, Germany \\ ${ }^{2}$ Department of Medicine II, Klinikum Rechts der Isar, Technische Universität München, 81675 \\ München, Germany \\ ${ }^{3}$ Institute of Laboratory Animal Science, University of Zurich, Zurich \\ ${ }^{4}$ Institute of Pathology, Technische Universität München, 81675 München, Germany \\ ${ }^{5}$ German Cancer Consortium (DKTK), 69120 Heidelberg, Germany \\ ${ }^{6}$ German Center for Infection Research (DZIF), partner site Munich, Germany
}

\begin{abstract}
T cell non-Hodgkin lymphomas (T-NHLs) represent a heterogeneous group of highly aggressive malignancies with poor clinical outcomes1. T-NHLs originate from peripheral T lymphocytes and are frequently characterized by genetic gain-of-function variants in T cell antigen receptor (TCR) signalling molecules1-4. Although these oncogenic alterations are thought to drive TCR pathways to induce chronic proliferation and survival programmes, it remains unclear whether $\mathrm{T}$ cells harbour tumour suppressors that can counteract these events. Using a murine model of human $\mathrm{T}$ cell lymphoma, we demonstrate that the acute enforcement of oncogenic TCR signalling in lymphocytes drives the strong expansion of these cells in vivo. However, this response is shortlived and robustly counteracted by cell-intrinsic mechanisms. A subsequent genome-wide in vivo
\end{abstract}

Users may view, print, copy, and download text and data-mine the content in such documents, for the purposes of academic research, subject always to the full Conditions of use:http://www.nature.com/authors/editorial_policies/license.html\#terms

Correspondence and requests for materials should be addressed to J.R. (j.ruland@tum.de).

Author Contributions. T.W. and J.R. designed the study. T.W. performed most of the experiments. Z.K. generated the data for the in vivo experiments with checkpoint inhibitors. S.K. performed intracellular flow cytometric and Phosflow analyses. K.P. contributed to in vivo experiments. E.H. performed experiments involving human cell lines and primary human cells. R.R. provided mouse lines and guided the transposon screen. R.Ö. and R.M. carried out the QIseq and TAPDANCE analysis. K.S. performed the pathohistological analyses. C.W. performed the bioinformatical analysis on human WGS and WES data. T.W., K.P. and C.W. generated the figures. J.R., T.W. and C.W. wrote the manuscript. All authors discussed the results and contributed to the manuscript.

Author Information. The authors declare no competing financial interests.

Data Availability. The sequencing data generated in this study have been deposited in the NCBI Sequence Read Archive (SRA) under accession PRJNA362826. The publicly available WGS/WES and RNAseq data from human patients are accessible in the European Genome-phenome Archive under accession EGAS00001001296 and in the SRA under accession PRJNA285408. RNAseq data from the publicly available dataset GSE8331534 in the Gene Expression Omnibus data repository was used as stated in the methods section. Source Data is provided for Figures 1a and c, 4a, b, c, d, f and h, and for the Extended Data Figures 1a and e, 2a and d, 6b and g, 7f and $\mathrm{g}$, and $8 \mathrm{~d}$. All other data are available as Supplementary Information, Source Data, or upon reasonable request from the corresponding author (J.R.). 
screen using T cell-specific transposon mutagenesis identified $P D C D 1$, which encodes the inhibitory receptor Programmed Death-1 (PD-1), as a master gene suppressing oncogenic $\mathrm{T}$ cell signalling. Mono- and bi-allelic PDCD1 deletions are also recurrently observed in human $\mathrm{T}$ cell lymphomas with frequencies that can exceed $30 \%$, indicating high clinical relevance.

Mechanistically, PD-1 activity enhances PTEN levels and attenuates AKT and PKC signalling in pre-malignant cells. In contrast, a homo- or heterozygous deletion of PD-1 allows unrestricted T cell growth after an oncogenic insult and leads to the rapid development of highly aggressive lymphomas in vivo that are readily transplantable to recipients. Altogether, these results indicate that the inhibitory PD-1 receptor is a potent haploinsufficient tumour suppressor in T-NHLs that is frequently altered in human disease. These findings extend the known physiological functions of PD-1 beyond the prevention of immunopathology after antigen-induced $\mathrm{T}$ cell activation and have implications for $\mathrm{T}$ cell lymphoma therapies and for current strategies that target PD-1 in the broader context of immuno-oncology.

Recent integrated molecular studies of human $\mathrm{T}$ cell lymphomas have identified activating mutations in signalling molecules that regulate $\mathrm{T}$ cell antigen receptor (TCR) pathways as a hallmark of most $\mathrm{T}$ cell non-Hodgkin lymphoma (T-NHL) subtypes1-6. These alterations affect antigen receptor proximal regulators, PI3K elements that engage the AKT pathway, mediators of antigen-induced NF- $\mathrm{kB}$ activation, such as PKCs and CARD11, and various other factors1-6. A specific chromosomal translocation that is recurrently detected in human peripheral $\mathrm{T}$ cell lymphoma cases is $\mathrm{t}(5 ; 9)(\mathrm{q} 33 ; \mathrm{q} 22) 7,8$, which fuses the antigen receptor kinase genes $I T K$ and $S Y K$. The chimeric ITK-SYK protein exhibits constitutive tyrosine kinase activity and enforces the oncogenic activations of TCR pathways via the activation of $\mathrm{PLC} \gamma, \mathrm{PI} 3 \mathrm{~K} / \mathrm{AKT}$ signalling and PKC/NF- $\mathrm{kB} 8$. We used this fusion protein to experimentally explore the pathomechanisms of $\mathrm{T}$ cell lymphomagenesis, a paradigm that has been successfully exploited in several other haematopoietic malignancies with recurrent chromosomal translocations9. To generate a genetically tractable model of human T-NHL, we had introduced a patient-derived ITK-SYK together with a eGFP cDNA into the murine Rosa26 locus preceded by a loxP-flanked STOP cassette (LSL; Rosa26 $6^{\text {LSL-ITK-SYK }}$ mice)8. Crossing Rosa26 $6^{\text {LSL-ITK-SYK }}$ mice to CD4-Cre transgenic mice for the T cell-specific ITKSYK/eGFP expression induced fully penetrant aggressive $\mathrm{T}$ cell lymphomas in the offspring (ITK-SYK ${ }^{\mathrm{CD} 4-\mathrm{Cre}}$ mice) that exhibited molecular, clinical and pathological features of the human disease8 (Extended Data Fig. 1a, b, c). Although the constitutively active CD4-Cre transgene drives continuous ITK-SYK expression in millions of polyclonal T cells, the final lymphomas are typically clonal (Extended Data Fig. 1d)8. In contrast to polyclonal T cells from young ITK-SYK ${ }^{\mathrm{CD} 4-\mathrm{Cre}}$ mice these clonal lymphoma cells transmit the disease to recipient mice (Extended Data Fig. 1e) indicating that they possess genetic alterations in addition to ITK-SYK expression, which promote malignancy.

To assess the evolution of these cancers in a controlled manner, we crossed Rosa26 $6^{\text {LSL-ITK-SYK }}$ mice with animals that allow tamoxifen-inducible Cre activation in $\mathrm{CD}^{+} \mathrm{T}$ cells $\left(\mathrm{CD} 4-\mathrm{CreER}{ }^{\mathrm{T} 2}\right.$ mice) 10 . We triggered single pulses of Cre activity in subsets of lymphocytes in the progeny (ITK-SYK ${ }^{\text {CD4-CreERT2 }}$ mice) (Fig. 1a, b, c). ITK-SYK and eGFP expression in individual lymphocytes led to a rapid expansion of these cells in vivo (Fig. 1a). The maximal frequencies of ITK-SYK ${ }^{+} \mathrm{CD}^{+} \mathrm{T}$ cells increased with increasing 
doses of tamoxifen $(\mathrm{r}=0.99)$. However, after this expansion phase, the ITK-SYK ${ }^{+}$ compartments again contracted (Fig. 1a). To characterize these two phases, we again induced ITK-SYK/eGFP expression in T cells in vivo and then FACS-sorted recombined $\mathrm{CD}^{+} \mathrm{T}$ cells for an in vitro RNAseq analysis (Fig. 1b). Gene set enrichment analysis (GSEA) revealed enrichment in the signatures Ishida_E2F_targets11, Hallmark_G2M_checkpoint12 and Whitfield_cell_cycle_literature13 in the ITK-SYKexpressing cells at day 4 compared with that of naïve $\mathrm{CD} 4^{+} \mathrm{T}$ cells demonstrating a highly proliferative phenotype. However, at day 7 , the proliferative signatures were significantly downregulated $(\mathrm{p}<0.01)$ along with the declining ITK-SYK ${ }^{+} \mathrm{T}$ cell numbers in vivo, indicating that $\mathrm{T}$ cells possess tumour-suppressive mechanisms that can counteract oncogenic T cell signalling within days. Nevertheless, within one year, two of three mice that received the highest dose of tamoxifen $(1 \mathrm{mg})$ developed ITK-SYK/eGFP-expressing $\mathrm{CD}^{+} \mathrm{T}$ cell lymphomas (Fig. 1c), with organ infiltration by malignant lymphoblasts (Extended Data Fig. 1f, g), which demonstrated that the tumour-suppressive pathways can be disrupted. Because the onset of these lymphomas was correlated with the initial frequency of the ITK-SYK-expressing T cells (Fig. 1c; $p=0.005$ ), these tumour-enabling secondary events are presumably stochastic in nature.

To reveal the mechanisms that enable full $\mathrm{T}$ cell transformation after oncogenically enforced TCR signalling we designed a genome-wide forward genetic screen (Fig. 2a) using the piggyBac (PB) transposition system for in vivo mutagenesis 14,15. To achieve spatially restricted transposition in only the ITK-SYK-sensitized T cells, we crossed Rosa26 $6^{\text {LSL-ITK-SYK }}$ to CD4-Cre mice and animals conditionally expressing the PB transposase from the Rosa26 locus (Rosa26 ${ }^{\mathrm{LSL}-\mathrm{PB}}$ ) and to ATP2-H32 transgenic mice harbouring mutagenic ATP transposon cassettes that could be mobilized by PB15,16. The quadruple-transgenic progeny developed accelerated $\mathrm{T}$ cell lymphomas after transposon mobilization (Extended Data Fig. 2a, b, c). The integration sites were recovered from FACSsorted $\mathrm{eGFP}^{+}$cancer cells by multiplexed insertion site sequencing (QISeq) 15 followed by statistical analyses 17 to identify the genomic regions that were more frequently hit by transposons than expected by chance (Fig. 2a). Remarkably, the top ranking common insertion site (CIS) (genomic region with the highest transposon insertion density) with the highest significance $\left(\mathrm{p}=0.87 \times 10^{-31}\right)$ across the genome was located at the Pdcd1 locus that encodes the inhibitory receptor Programmed Death-1 (PD-1)18 (Fig. 2b and Extended Data Fig. 2e). These insertions did not co-exist with common insertions in other loci and resulted in Pdcd1-transposon fusion transcripts (Extended Data Fig. 2f). Some cancers had multiple $P d c d 1$ insertions that most likely reflected bi-allelic inactivation and/or local transposon hopping with gene trapping in subclones. Lymphomas with $P d c d 1$ transposon insertions exhibited reduction in or lack of PD-1 protein and Pdcd1 mRNA expression (Fig. 2c and Extended Data Fig. 2d).

PD-1 functions to restrict antigen-induced TCR responses at tolerance checkpoints with persistent antigenic stimulation for the prevention of autoimmunity 18 and has received considerable attention because it limits antigen-driven $\mathrm{T}$ cell proliferation and survival in suppressive microenvironments in cancer and chronic infection19. Our genetic screen indicated that PD-1 might also possess unrecognized functions as a tumour suppressor in $\mathrm{T}$ cell lymphoma. To study potential PDCD1 alterations in human T cell lymphoma, we 
performed a meta-analysis of five published studies2-6. Strikingly, we found genomic PDCD1 alterations in 36 of 158 cases (23\%) (Extended Data Table 1). The predominant mutation type was copy number aberration (33 cases). Among these, focal deletions with mono- or bi-allelic PDCD1 loss were prominent (Fig. 2d, Extended Data Table 1 and Extended Data Fig. 3a, b). The highest proportion of homo- or heterozygous PDCD1-deleted cases $(36 \%, 13$ out of 36$)$ was found in a group of patients with advanced stage IV cutaneous T cell lymphoma3 (Extended Data Table 1). A single case with PDCD1 genomic gain exhibited defective $P D C D 1$ mRNA expression (Extended Data Fig. 3a). In light of our genetic screen, these frequent homo- or heterozygous PDCD1 deletions in human T-NHL strongly suggest that PD-1 is functionally relevant in lymphoma pathogenesis.

In normal T cells, the PD-1 expression is upregulated upon TCR ligation19 and PD-1 subsequently provides feedback for the prevention of autoimmunity. To investigate whether oncogenic T cell signals can also induce PD-1 expression, we introduced ITK-SYK variants into Jurkat T cells8. ITK-SYK signalling drives PD-1 expression in a kinase-dependent manner (Extended Data Fig. 4a). In transduced primary human $\mathrm{CD}^{+}{ }^{+} \mathrm{T}$ cells, we also detected the ITK-SYK kinase-mediated upregulation of PD-1 expression (Extended Data Fig. 4b). Likewise, acute ITK-SYK expression in primary $\mathrm{CD} 4^{+} \mathrm{T}$ cells from tamoxifentreated ITK-SYK ${ }^{\mathrm{CD} 4-C r e E R T 2}$ mice drives also robust PD-1 protein and mRNA expression (Fig. 3a, b and Extended Data Fig. 4c). Moreover, the analysis of human T-NHL samples $(\mathrm{n}=50) 2,6$, which did or did not exhibit the activation of the gene signature GO_antigen_receptor_mediated_signaling_pathway (195 genes) demonstrated that the aberrant activation of TCR signalling pathways was correlated with significantly upregulated PDCD1 mRNA expression (Fig. 3c, d) in these tumours. In addition, within this patient group, loss of one or two $P D C D 1$ alleles led to a significantly reduced or completely absent $P D C D 1$ mRNA expression (Fig. 3e). Thus, $P D C D 1$ expression is upregulated after oncogenic T cell signalling in murine and human cells, and the loss of the PDCD1 locus in human T-NHL impairs PDCD1 mRNA expression.

PD-1 ligation dampens physiological antigen-mediated $\mathrm{T}$ cell activation by enhancing the tumour suppressor phosphatase PTEN20 and attenuating the PI3K/AKT21 and PKC $9 / \mathrm{NF}-$ $\mathrm{B}$ pathways 22. Because these two cascades are critical for lymphomagenesis, we tested whether they were sensitive to PD-1 ligation after oncogene induction. We activated ITKSYK in vivo by injecting tamoxifen into ITK-SYK ${ }^{\mathrm{CD} 4-\mathrm{CreERT} 2}$ mice, isolated $\mathrm{CD} 4^{+}$cells, stimulated them with PD-L1 and subsequently performed an intracellular Phosflow analysis. The engagement of PD-1 increased PTEN protein levels and attenuated the AKT and PKC $\theta$ activities in pre-malignant ITK-SYK expressing cells (Fig. 3f). Thus, oncogenic T cell signalling upregulates PD-1 expression and PD-1 functions to suppress oncogenic effector pathways.

To study the oncogene-PD-1 loop in tumour suppression in vivo, we crossed the Rosa26 $6^{\text {LSL-ITK-SYK }}$ and CD4-CreER ${ }^{\text {T2 }}$ alleles on a PD-1-deficient background 23 and triggered ITK-SYK expression via tamoxifen in the offspring. As previously observed, the marked expansion of ITK-SYK $/ \mathrm{eGFP}^{+} \mathrm{T}$ cells was robustly counter-regulated in the presence of PD-1 (Fig. 4a). In sharp contrast, without PD-1, the ITK-SYK ${ }^{+}$T cells expanded without restriction, and all tamoxifen-treated ITK-SYK ${ }^{\mathrm{CD} 4-\mathrm{CreERT} 2} ; \mathrm{PD}-1^{-/-}$animals had to 
be euthanized after only one week because of a strong accumulation of lymphoblastoid ITK$\mathrm{SYK}^{+} \mathrm{PD}-1^{-/-} \mathrm{CD} 4^{+} \mathrm{T}$ cells in the lymphoid tissues and invasive growth with a noncohesive pattern into the solid organs (Fig. 4a and Extended Data Fig. 5a, b, c). Upon transplantation these ITK-SYK ${ }^{+/} \mathrm{eGFP}^{+} \mathrm{PD}-1^{-/-} \mathrm{CD} 4^{+} \mathrm{T}$ cells continued to expand invasively and transmitted the lethal disease to recipients demonstrating that these cells were fully transformed malignant lymphocytes (Fig. 4b and Extended Data Fig. 5d, e, f, g). In parallel, we incubated $\mathrm{CD}^{+}{ }^{+} \mathrm{T}$ cells from Rosa26 $6^{\mathrm{LSL}-I T K-S Y K}$ and Rosa26 $6^{\mathrm{LSL}-I T K-S Y K} ; \mathrm{PD}-1^{-/-}$mice without Cre transgenes with the recombinant TAT-CRE fusion recombinase24 in vitro and injected $5 \times 10^{5}$ treated cells into syngeneic C57BL/6 wild-type mice, which had not received any pre-conditioning treatment (Extended Fig. 6a, b, c, d, e, f). The TAT-CRE-treated ITKSYK-expressing $\mathrm{CD}^{+}{ }^{+} \mathrm{T}$ cells from PD-1-competent donors (Rosa26 $6^{\mathrm{LSL}-I T K-S Y K}$ ) disappeared over time (Extended Data Fig. 6c). In contrast, those from PD-1-deficient

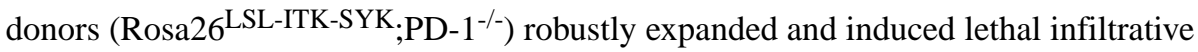
lymphomas that could be serially transplanted into syngeneic animals (Extended Data Fig. $6 \mathrm{~g}, \mathrm{~h}, \mathrm{i}, \mathrm{j})$. Thus, the absence of PD-1 in peripheral T cells enables an appropriate oncogenic signal to directly create an aggressive cancer.

Because mono-allelic deletions are the most common type of $P D C D 1$ alteration in human $\mathrm{T}$ NHL (see Fig. 2d, Extended Data Table 1 and Extended Data Fig. 3b), we also treated heterozygous ITK-SYK ${ }^{\mathrm{CD} 4-\mathrm{CreERT}} ; \mathrm{PD}-1^{+/-}$mice with tamoxifen. Intriguingly, all heterozygous ITK-SYK ${ }^{\mathrm{CD} 4-\mathrm{CreERT}}$;PD-1 ${ }^{+/-}$mice succumbed to invasive transplantable lymphomas after tamoxifen injection (Fig. 4c and Extended Data Fig. 7a, b, c, d, e, f, g, h, i, j), establishing PD-1 as a haploinsufficient tumour suppressor.

The inhibition of the PD-1/PD-L1 interaction with antibodies against PD-1 or PD-L1 is heavily exploited in the clinic to activate $\mathrm{T}$ cell immunity against multiple cancer cell

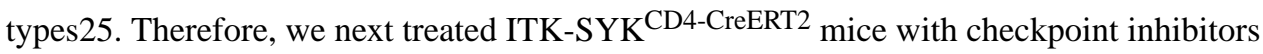
(anti-PD-L1 or anti-PD-1) after tamoxifen induction (Fig. 4d, e, f and Extended Data Fig. 8a, b, c, e). Strikingly, similar to a genetic PD-1 deletion, anti-PD-L1 treatment beginning one day after tamoxifen induction lead to an immediate invasive ITK-SYK ${ }^{+} \mathrm{T}$ cell proliferation (Fig. 4d, e and Extended Data Fig. 8a, b, c). Likewise, treatment of ITK$\mathrm{SYK}^{\mathrm{CD}} 4-\mathrm{CreERT} 2$ mice with anti-PD-L1 or anti-PD-1 antibodies during the contraction phase of the ITK-SYK ${ }^{+}$cell compartment (ten days after tamoxifen treatment) also induced lethal lymphoproliferation (Fig. 4f and Extended Data Fig. 8e). However, these cells were unable to transmit the disease when transplanted into non-antibody-treated recipients (Extended Data Fig. 8d). Thus, although these checkpoint inhibitors accelerate the growth of $\mathrm{T}$ cells with oncogenically sensitized TCR pathways, their pharmacological effects are only transient in contrast to the effect of T cell intrinsic Pdcd1 gene deletion.

We demonstrated above that the ligation of PD-1 enhances PTEN levels and attenuates PI3K/AKT and PKC $\theta$ function after ITK-SYK signalling (see Fig. 3f). PD-1 signalling in a PD-1-transduced human lymphoma cell line also enhances PTEN and attenuates PI3K/AKT and PKC $\theta$ (Extended Data Fig. 9a, b, c). Consistently, in murine PD-1-deficient ITK-SYK ${ }^{+}$ lymphomas, PTEN levels are reduced and AKT and PKC $\theta$ activity is enhanced (Fig. 4g), suggesting that $P D C D 1$ deletions promote tumourigenesis, at least in part, via these mechanisms. To study the functional relevance of PI3K/AKT enforcement, we incubated 
PD-1-deficient lymphoma cells in vitro with the PI3K inhibitor idelalisib, which is currently undergoing clinical development for selected B cell malignancies26. PI3K inhibition downregulated AKT activity and induced death of ITK-SYK ${ }^{+}$PD-1 ${ }^{-/-}$lymphoma cells (Extended Data Fig. 9d). Finally, we treated mice that harbour ITK-SYK ${ }^{+} \mathrm{PD}-1^{-/-}$ lymphomas with idelalisib in vivo. PI3K inhibition significantly extended the lifespan of these mice, demonstrating that the activity of PI3K/AKT signalling is critical for lymphomas with Pdcd1 deletion (Fig. 4h and Extended Data Fig. 9e).

In conclusion, our studies establish PD-1 as a powerful haploinsufficient tumour suppressor in T cell lymphoma. Thus, PD-1 not only functions to prevent autoimmunity after chronic antigen exposure and physiological TCR ligation but also unexpectedly counteracts oncogenic T cell signalling pathways within precancerous cells. The deficiency in PD-1 in T cell lymphoma promotes PI3K/AKT activity, and our preclinical data suggest PI3K inhibitors as candidate drugs for the treatment of these tumours. Checkpoint blockade with anti-PD-1 or anti-PD-L1 to relieve T cell inhibition in tumour microenvironment27 is a revolutionary treatment of multiple malignancies 25 and is currently being explored in TNHL. Our findings that these checkpoint inhibitors can accelerate and/or reactivate $\mathrm{T}$ cell clones with oncogenically activated TCR pathways indicate the need for special consideration when using these in T-NHL patients. In addition, new clinical trials using CRISPR/Cas9-mediated gene editing to bi-allelically disrupt the PDCD1 locus in peripheral $\mathrm{T}$ cells of cancer patients are being initiated with the goal of enhancing anti-tumour immune responses28. Reports have suggested that these treatments could trigger autoimmune reactions28. Our data demonstrating that a suitable oncogenic hit on PD-1-deficient $\mathrm{T}$ cells is sufficient for their direct malignant transformation puts additional caution on these gene editing approaches.

\section{Methods}

\section{Mice}

Mice of both sexes aged 6-12 weeks were used for all experiments. Littermate controls were used whenever possible. Randomization and blinding were not performed.

Rosa26 $6^{\text {LSL-ITK-SYK }}$ mice8 were backcrossed for more than 12 generations on a C57BL/6 genetic background. CD4Cre-ER ${ }^{\mathrm{T} 2} 10$ mice were crossed to the Rosa26 $6^{\mathrm{LSL}-\mathrm{ITK}-\mathrm{SYK}}$ strain to generate the ITK-SYK ${ }^{\mathrm{CD}} 4 \mathrm{Cre}-\mathrm{ERT} 2$ mice. Rosa26 $6^{\mathrm{LSL}-\mathrm{PB}}$ mice16 were crossed to Rosa26 $6^{\text {LSL-ITK-SYK }}$ mice to generate the Rosa26 $6^{\text {LSL-ITK-SYK;Rosa26 }}{ }^{\text {LSL-PB }}$ strain, and ATP2-H3214 mice were crossed to CD4-Cre mice to generate the ATP2;CD4-Cre transgenic animals. Both resulting lines were crossed again to generate the quadruple-transgenic

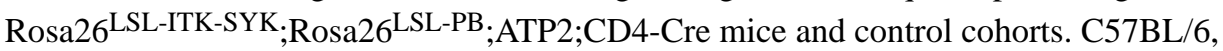
NOD/SCID Il2 $\mathrm{rg}^{-/-}$(NSG, 005557), CD4-Cre (02207129) and PD-1/- (02827623) mice were purchased from the Jackson Laboratory, Bar Harbor, Maine, USA. CD4-Cre and PD- $1^{-/-}$mice were backcrossed on a C57BL/6 genetic background. All animal experiments were performed according to local guidelines (Regierung von Oberbayern, Munich, Germany). Mice were euthanized if they exhibited signs of lymphoma (lymph node enlargement, palpable tumour, laboured breathing, ascites) or if they lost $20 \%$ or more of their body weight. None of the approved thresholds were exceeded at any time. 


\section{Flow cytometry}

Flow cytometric analyses were performed using standard protocols. Primary cell suspensions from the spleen, lymph nodes, or peripheral blood were directly labelled. Single-cell suspensions from the liver, kidney, or lung were first purified by Percoll (17089101, GE Healthcare, Chicago, Illinois, USA) gradient centrifugation. Fluorescently labelled antibodies against the following surface proteins were used for staining mouse cells: TCR $\beta$ (APC/Cy7-labeled anti-mouse TCR $\beta$ chain antibody, 10922), CD4 (Pacific bluelabelled anti-mouse CD4 antibody, 100428), CD44 (PE-labelled anti-mouse/human CD44 antibody, 103008), and PD-1 (APC-labelled anti-mouse CD279 (PD-1) antibody, 135210). For the human cells, the following antibodies were used: PD-1 (APC-labelled anti-human CD279 (PD-1) antibody, 329908), CD3 (PE/Cy7-labelled anti-human CD3 antibody, 300420) and PD-L1 (PE-labelled anti-human CD274 (B7-H1, PD-L1) antibody, 329706). Fc receptor blocking was performed with anti-CD16/32 antibodies (101320 or 422302). All antibodies were purchased from BioLegend, San Diego, California, USA. The data were acquired using a FACSCanto II flow cytometer (Becton, Dickinson and Company, Franklin Lakes, New Jersey, USA). FlowJo software (FlowJo LLC, Ashland, Oregon, USA) was used for data analyses.

\section{GeneScan analysis of TCR gene rearrangements}

Genomic DNA was extracted (DNeasy, Qiagen, Venlo, The Netherlands) from magnetically purified (130104454, Miltenyi Biotec, Bergisch Gladbach, Germany) CD4+ T cells from

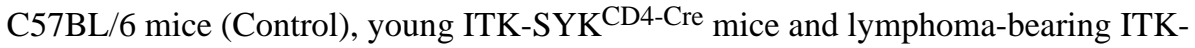
SYK $^{\mathrm{CD} 4-C r e}$ mice. GeneScan analysis to detect TCR gene rearrangements was performed as previously described8. In summary, VDJ rearrangements of the TCR $\beta$ and TCR $\gamma$ loci were amplified via five PCR reactions with 26 primers in total, with three primers labelled with 5'-FAM fluorochromes. Fluorochrome-labelled PCR products were then size-separated and scanned via capillary electrophoresis.

\section{Induction of spontaneous ITK-SYK expression in peripheral T cells}

Tamoxifen (T5648, Sigma-Aldrich, St. Louis, Missouri, USA) was dissolved in pure ethanol (with heating) and subsequently diluted in migliol (3274, Caesar \& Loretz, Hilden, Germany) to a final concentration of $10 \mathrm{mg} / \mathrm{ml}$. To induce the expression of ITK-SYK in a small fraction of peripheral CD4 ${ }^{+} \mathrm{T}$ cells, ITK-SYK ${ }^{\mathrm{CD} 4-C r e E R T 2}$, ITK$\mathrm{SYK}^{\mathrm{CD} 4-\mathrm{CreERT}} ; \mathrm{PD} 1^{+/-}$or ITK-SYK ${ }^{\mathrm{CD} 4-\mathrm{CreERT}} ; \mathrm{PD}-1^{-/-}$mice were given tamoxifen at the indicated doses via intraperitoneal (i.p.) injection.

\section{RNA}

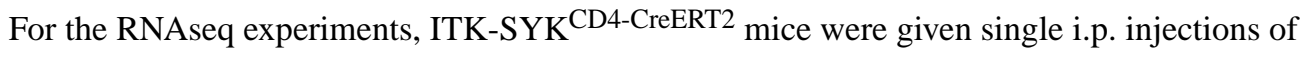
$1 \mathrm{mg}$ tamoxifen per mouse (T5648, Sigma-Aldrich). Forty-eight hours after injection, the spleens were harvested; the single-cell suspensions were subjected to $\mathrm{CD} 4^{+} \mathrm{T}$ cell purification by magnetic depletion (130104454, Miltenyi Biotec), and the cells were then cultured in vitro. At the indicated time points, the T cells were FACS-sorted (FACSAria, Becton, Dickinson and Company) again for viability and eGFP expression. Total RNA was extracted from approximately 100,000 cells using spin columns (RNeasy Micro Plus Kit, 
Qiagen). Library preparation was performed from $1 \mathrm{ng}$ total RNA with the SMART-Seq v2 Ultra Low Input RNA Kit (Takara Bio Inc., Kusatsu, Shiga, Japan), and SE-50 bp sequencing was performed on Illumina HiSeq2000 machines (Illumina, San Diego, California, USA). Read alignment to the $\mathrm{mm} 10$ genome and transcriptome assembly were performed using HISAT230 and StringTie31, respectively. Differential expression was assessed with Ballgown32. GSEA was performed with BubbleGUM33; to assess proliferation, the following signatures were selected: Whitfield_cell_cycle_literature13, Ishida_E2F_targets11 and Hallmark_G2M_checkpoint12. RNAseq data for naïve murine $\mathrm{CD}^{+} \mathrm{T}$ cells were retrieved from the Gene Expression Omnibus data repository series GSE8331534 (samples 83-85), re-aligned to the mm10 genome using HISAT2 and then processed in a manner similar to that used for the other samples.

For Pdcd1 mRNA quantification within lymphoma cells from Rosa26 $6^{\text {LSL-ITK-SYK; }}$;osa26 $6^{\text {LSL-PB }}$;ATP2;CD4-Cre mice, total RNA was extracted from $\mathrm{eGFP}^{+}$FACS-sorted spleen-derived lymphocytes using spin-columns and then reverse transcribed (M0368L, NEB, Ipswich, Massachusetts, USA). The Pdcd1 and eGFPcDNA was simultaneously quantified using transcript-specific probes (4331182, Mm01285676_m1, FAM-MGB and 4448484, Mr03989638_mr, VIC-MGB_PL, Thermo Fisher Scientific, Waltham, Massachusetts, USA) on a droplet digital PCR system (QX200 Droplet Digital PCR System, 1864001, Bio-Rad, Hercules, California, USA). Subsequently, the amount of $e G F P$-normalized $P d c d 1$ cDNA was categorized based on the presence of transposon cassettes within the Pdcd1 locus.

The primers for the identification of the Pdcd1-transposon mRNA splice junctions are listed in Supplementary Table 1.

\section{Quantitative insertion-site sequencing and identification of common integration sites}

Lymphoma cells from Rosa26 $6^{\text {LSL-ITK-SYK;Rosa26 }}{ }^{\text {LSL-BP;ATP2;CD4-Cre mice were }}$ harvested by generating single-cell suspensions from the spleens and lymph nodes and then sorting for $5 \times 10^{6}$ viable $\mathrm{eGFP}^{+} \mathrm{CD} 4^{+} \mathrm{T}$ cells. Genomic DNA was extracted using spin columns. The transposon insertion-sites were recovered using QiSeq, a splinkerette-PCRbased method for semi-quantitative insertion-site sequencing36. Bioinformatics data processing, mapping of sequence reads and statistical analyses to identify CIS using TAPDANCE were performed as described earlier36. The CISs that were also detected in the data from control animals without the ITK-SYK transgene were excluded from analysis.

\section{Genomic PDCD1 alterations in human T cell lymphoma}

PubMed and Google Scholar were searched for published studies on human T cell malignancies that included WGS, WES, or targeted sequencing data. The supplementary data from the identified studies were searched for single nucleotide variants, small indels, and copy number aberrations (CNAs) that affect the PDCD1 locus. Whole-genome sequencing data (aligned BAM files, 47 tumour-normal pairs) 2 were downloaded from the European Genome-phenome Archive with permission. Whole exome sequencing data (aligned BAM files, 34 tumour-normal pairs)6 were downloaded from the Sequence Read Archive (accession PRJNA285408). The BAM files were subjected to a copy number 
analysis using Control-FREEC36. The copy numbers were calculated for each sample in windows of $3 \mathrm{~kb}$ with steps of $1 \mathrm{~kb}$ using tumour and corresponding normal samples as inputs. Breakpoint analysis, segmentation and absolute copy number calling were performed with Control-FREEC. The resulting segments and copy number ratios (tumour/normal) were transformed to a $\log 2$ scale and plotted for all tumours along chromosome 2 for visual inspection.

\section{GSVA and PDCD1 FPKM analyses in human T cell lymphoma}

RNAseq data of human T-NHL patients were downloaded from the Sequence Read Archive (accession PRJNA285408)6 and the European Genome-phenome Archive2. Reads were extracted in fasta format from sra or bam files and aligned to the hg19 genome with HISAT230 and processed with StringTie31 and Ballgown32. All samples with available genomic data (WES or WGS) were included in the analysis. Using GSVA37 for each sample, an enrichment score was calculated based on the gene set GO_antigen_receptor_mediated_signaling_pathway (195 genes) from the MSigDB database: Twenty-eight of fifty samples that were identified as positive for the signature were included in the subsequent analysis. FPKM values for PDCD1 were extracted and statistically compared based on the enrichment score and the PDCD1 copy number status as indicated in the figure legend.

\section{Cloning}

To generate the PD-1 vector, first, the 3' LTR of the lentiGuide-Puro Plasmid (52963, Addgene, Cambridge, Massachusetts, USA) was replaced by an HIV/MSCV hybrid LTR as described38. The LTR-modified backbone and the central polypurine tract (cPPT) sequence was then amplified with the BsmBI restriction site-containing oligos BBf, BBr or CPPTf and CPPTr. Finally, the PDCD1 cDNA was cloned together with an IRES-GFP and a cPPT sequence into the modified backbone via Golden Gate Assembly using the PDCD1f and PDCD1r primers for the cDNA and the IRESGFPf and IRESGFPr primers for the IRESGFP sequence. The PD-1 YFYF plasmid was subsequently generated using sequential sitedirected mutagenesis with the $\mathrm{Y} 223 \mathrm{~F}$ and $\mathrm{Y} 248 \mathrm{~F}$ primer pairs. The oligo sequences are listed in Supplementary Table 2.

\section{Cell culture}

T cells were cultured in RPMI- 1640 medium containing $20 \%$ FCS in the case of primary cells, $10 \%$ FCS in the case of HuT 78 T-NHL cells 39 or 5\% FCS in the case of Jurkat-Eco T cells. To culture the Phoenix-Eco and Phoenix-Ampho packaging cells, we used DMEM with 10\% FCS. HEK293FT cells were cultured in 5\% FCS containing DMEM. The HuT 78 cell line was authenticated at the DSMZ via STR profiling (Leibniz Institute DSMZ-German Collection of Microorganisms and Cell Cultures, Braunschweig, Germany). Otherwise, no authentication of the cell lines was performed. All cell lines were routinely tested for mycoplasma infection.

For the generation of dendritic cells, Ficoll (Ficoll-Paque PLUS, 17144002, GE Healthcare) gradient-separated PBMCs from healthy donors were magnetically enriched for monocytes (130050201, Miltenyi Biotec). Next, the cells were cultured for six days in the presence of 
recombinant IL-4 (20 ng/ml, 20004, Peprotech, Rocky Hill, New Jersey, USA) and GM-CSF $(100 \mathrm{ng} / \mathrm{ml}, 30003$, Peprotech). Finally, differentiated dendritic cells were induced to express PD-L1 with a 24-hours Interferon- $\gamma$ stimulation (100 ng/ml, 30002, Peprotech).

All human PBMCs were collected from healthy donors with informed consent according to the protocols approved by the Institutional Review Boards. The study protocol was approved by the local ethics committee (Ethics Committee of the Medical Faculty, Klinikum rechts der Isar, Technical University of Munich, Germany).

For the in vitro experiments using idelalisib (CAL-101, GS-1101, PI3Kס inhibitor; S2226, Selleckchem, Houston, Texas, USA), the inhibitor was dissolved in DMSO, and the cell culture medium was supplemented with the indicated concentration of the compound or DMSO (as a control).

\section{Retroviral infections}

Generation of ecotropic viral particles containing GFP, ITK-SYK or ITK-SYK ${ }^{\mathrm{KD}}$ constructs and infection of Jurkat-Eco cells were performed as previously described8. To generate amphotropic retrovirus, Phoenix-Ampho cells were grown in $15 \mathrm{~cm}$ dishes and transfected using FuGENE (Promega, Madison, Wisconsin, USA) with $10 \mu \mathrm{g}$ ITK-SYK or ITK-SYK KD 8 . The media was changed 8 hours after transfection. The viral supernatant was collected 36 hours after transfection, passed through a $0.45 \mu \mathrm{m}$ filter and used fresh or snap-frozen for later. Activated primary human CD4 T-cells were spinfected with RetroNectin-coated (Takara Bio Inc.) 6-well plates $(5 \mathrm{mg} / \mathrm{ml})$. For the production of lentiviral particles, we used HEK293FT cells. FuGENE-based transfection was performed with $17 \mu \mathrm{g}$ pCMV delta R8.2, $10 \mu \mathrm{g}$ pHCMV-10A1 and $10 \mu \mathrm{g}$ PD-1 or PD-1 YFYF. HH cells were spinfected with the lentivirus on RetroNectin-coated 6-well plates $(5 \mathrm{mg} / \mathrm{ml})$.

\section{Primary human T-cell isolation}

Human PBMCs were isolated by Ficoll gradient centrifugation of fresh blood obtained from healthy donors. CD4 T-cells were enriched by magnetic depletion (130096533, Miltenyi Biotec) and stimulated with immobilized anti-CD3 antibody $(10 \mu \mathrm{g} / \mathrm{ml}$, purified anti-human CD3 antibody, 300332, BioLegend), soluble anti-CD28 antibodies (2 $\mu \mathrm{g} / \mathrm{ml}$, purified antihuman CD28 antibody, 302934, BioLegend) and 10 ng/ml IL-2 (20002, Peprotech). After 48 hours, the cells were infected with retroviruses. Five days later, the T-cells were stimulated again with $2 \mu \mathrm{g} / \mathrm{ml}$ soluble anti-CD28 (302934, BioLegend) antibodies and analysed for PD-1 expression by flow cytometry.

\section{Transplantation experiments}

For ITK-SYK ${ }^{\mathrm{CD} 4-C r e}$ transplantation experiments, splenocytes from donor mice were harvested and single-cell suspensions were FACS-sorted for eGFP expression. Subsequently, $3 \times 10^{6}$ sorted cells were intravenously injected into C57BL/6 recipient mice.

For transplantation experiments into NSG mice, splenocytes from donor mice were harvested on day 5 or 7 after tamoxifen induction, subjected to CD8 T cell depletion (11447D, Thermo Fisher Scientific) and then i.v.-injected into NSG mice. 
For transplantation experiments without Cre transgenes, splenocytes from Rosa26 ${ }^{\text {LSL-ITK-SYK }}$ and Rosa26 ${ }^{\text {LSL-ITK-SYK} ; P D-1 /-~}$ mice were harvested, and the CD4 ${ }^{+} \mathrm{T}$ cells were purified and subsequently incubated for one hour in serum-free media (31985062, Thermo Fisher Scientific) supplemented with $1 \mu \mathrm{M}$ recombinant TAT-CRE protein (EG1001, Excellgen Inc., Rockville, Maryland, USA). After extensive washing, the cells were intravenously injected into C57BL/6 recipients. For transplantation into secondary C57BL/6 recipient mice, the spleens from diseased primary recipients were harvested, FACS-sorted for eGFP expression, washed and intravenously injected ( $1 \times 10^{5}$ cells/recipient) into wild-type C57BL/6 mice.

\section{In vivo $\mathrm{PI3K}$ inhibitor treatment}

For PI3K inhibitor treatment, recipient NSG mice were injected with $5 \times 10^{4} \mathrm{eGFP}^{+} \mathrm{T}$-cells together with CD8-depleted (11447D, Thermo Fisher Scientific) splenocytes from ITK$\mathrm{SYK}^{\mathrm{CD} 4-\mathrm{CreERRT2}}$;PD-1 ${ }^{-/-}$mice that had been treated with tamoxifen $(0.25 \mathrm{mg})$ five days earlier. Five days after transplantation, the NSG mice were given idelalisib (S2226, Selleckchem) or vehicle ( $0.5 \%$ carboxymethylcellulose, $0.05 \%$ Tween 80 in ultra-pure water, C5678 and P4780, Sigma-Aldrich) by oral gavage (10 mg per kg per day) for 5 days a week.

\section{Phosflow}

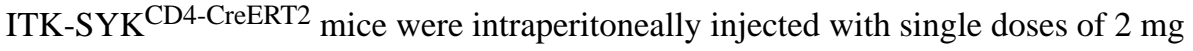
tamoxifen (TAM) per mouse. Forty-eight hours post-injection, single-cell suspensions were generated from the spleens and lymph nodes; then, $\mathrm{CD} 4^{+} \mathrm{T}$ cells were magnetically purified (130104454, Miltenyi Biotec), and eGFP expression was assessed by flow cytometry. The cells were stimulated with recombinant PD-L1-Fc chimera protein-coated (758208, BioLegend) microspheres (CS01N, Bangs Laboratories Inc., Fishers, Indiana, USA). In brief, streptavidin-coated microspheres were first incubated with a saturating amount of biotinylated anti-human IgG antibody (409302, BioLegend) for one hour at $37^{\circ} \mathrm{C}$ according to the manufacturer's instructions. After extensive washing, these pre-coated microspheres were loaded with either PD-L1-Fc chimeric protein or control recombinant human Fc fragments (AG714, Merck Group, Darmstadt, Germany). Efficient coating was ensured by flow cytometry. After 48 hours, the cells were fixed in $4 \%$ PFA followed by methanol permeabilization, stained for p-AKT (S473, 48-9715-42, Thermo Fisher Scientific), p-PKC $\theta$ (700043, Thermo Fisher Scientific) and PTEN (9550, Cell Signaling Technology Inc., Danvers, Massachusetts, USA) and assessed by flow cytometry. The following antibodies were used as secondary antibodies: PE Donkey anti-rabbit IgG (406421, BioLegend) or PE Goat anti-mouse IgG (405307, BioLegend).

To assess PTEN, p-AKT and p-PKC $\theta$ levels in ITK-SYK ${ }^{+}$PD- $1^{-/-}$lymphoma cells, TATCRE-treated Rosa26 $6^{\text {LSL-ITK-SYK }}$ and Rosa26 $6^{\text {LSL-ITK-SYK}} ; \mathrm{PD}-1^{-/-}$cells were isolated from C57BL/6 recipient mice (see Extended Data Fig. 6b). The single-cell suspensions were then stained for PTEN, p-AKT and p-PKC $\theta$ and analysed as above.

To analyse PTEN, p-AKT and p-PKC $\theta$ levels in the PD-1- or PD-1 YFYF-transduced TNHL cells, the $\mathrm{GFP}^{+}$FACS-sorted cells were incubated with equal amounts of PD-L1- 
expressing dendritic cells for 24 hours. Afterwards, the cells were fixed and permeabilized, stained for CD3, PTEN, p-AKT and p-PKC $\theta$ and then analysed as before.

To analyse the effects of idelalisib (S2226, Selleckchem) on AKT phosphorylation in vivo, $5 \times 10^{4}$ lymphoma cells from TAM-treated $(0.25 \mathrm{mg})$ ITK-SYK ${ }^{\text {CD4-CreERT2 }}$;PD- ${ }^{-/-}$animals were transferred into NSG recipient mice at day five after TAM injection. Five days later, the NSG mice were orally given a single dose of $10 \mathrm{mg} / \mathrm{kg}$ idelalisib or vehicle $(0.5 \%$ carboxymethylcellulose, $0.05 \%$ Tween 80 in ultra-pure water). Four hours later, splenic cells were extracted from the NSG mice, fixed and permeabilized in methanol, stained for p-AKT and analysed as above.

\section{In vivo checkpoint inhibition}

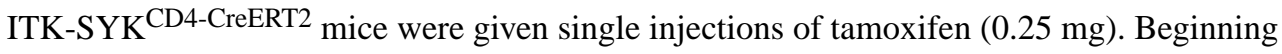
twenty-four hours later, the mice were given $200 \mu \mathrm{g}$ of anti-PD-L1 antibody (BE0101, Bioxell, West Lebanon, New Hampshire, USA) or $200 \mu \mathrm{g}$ anti-IgG2b (BE0090, Bioxcell) control antibody diluted to $200 \mu \mathrm{l}$ with PBS every second day. For the experiments in which the antibody treatment was started during the contraction phase, the ITK-SYK ${ }^{\text {CD4-CreERT2 }}$ mice were given $200 \mu \mathrm{g}$ of anti-PD-L1 or anti-PD-1 (BE0146, Bioxcell) antibody or antiIgG2b (BE0090, Bioxcell) control antibody diluted to $200 \mu$ with PBS every third day. In all experiments, the mice were monitored by flow cytometry of the peripheral blood.

\section{Histology}

All tissues were fixed in 4\% formaldehyde and paraffin embedded. Approximately 3-5 $\mu \mathrm{m}$ thick sections were cut and stained with H\&E. Immunohistochemistry was performed on an automated immunostainer (Agilent Technologies, Santa Clara, California, USA) using antibodies against CD3 (CI597C002, DCS, Hamburg, Germany) and Ki-67 (ab15580, Abcam, Cambridge, UK). The stained slides were evaluated by an experienced certified pathologist (K.S.) using a BX53 stereomicroscope (Olympus Corp., Shinjuku, Tokyo, Japan), classified according to the classification of lymphoid neoplasms in mice40 with respect to the most recent immunohistochemical phenotyping of rodent lymphoid tissues 41 and scanned using a slide scanner (AT-2, Leica Biosystems, Wetzlar, Germany). Representative images were collected using Aperio Imagescope software (version 12.3, Leica Biosystems).

\section{Statistics}

All statistical tests were performed using R3.1.3 software (R Foundation for Statistical Computing). In each experiment, the appropriate statistical tests, including non-parametric tests, were used as indicated in the figure legends. Multiple comparisons were performed with ANOVA, Tukey's post-hoc test and corrected p-values. To assess the correlation between the eGFP maxima and the initial tamoxifen doses (Fig. 1a), the local maxima (reached on day 4 for the $1 \mathrm{mg}$ and $0.25 \mathrm{mg}$ doses and on day 8 for the $0.05 \mathrm{mg}$ dose of tamoxifen) of the mean $\mathrm{eGFP}^{+}$cell frequencies were used for Pearson coefficient calculations. The association between lymphoma development and initial $\mathrm{eGFP}^{+}$cell frequency (Fig. 1c) was calculated based on the pooled data from two experiments. Fisher's exact test was performed on a $2 \times 2$ contingency table that was created based on two 
categorical variables: "lymphoma development" and "eGFP maximum $>5 \%$ during initial expansion phase". The variances within the groups in Fig. 1c were significantly different as determined by F-test. The normalized enrichment score (NES), false discovery rate (FDR) and $p$ values for the GSEA were calculated with BubbleGUM33. The p-values for the common integration sites (CISs) of the PB transposon cassettes were calculated with TAPDANCE17; the p-values of "insertions" were used for CIS ranking. The upper tail of the probability density function in Fig. $2 b$ was calculated based on the Poisson rate parameter $\lambda$ derived from TAPDANCE and the Poisson cumulative distribution function (ppois) in R. No statistical methods were used to predetermine sample size estimates. $\mathrm{P}<0.05$ was considered statistically significant; for the log-rank test using more than two groups, the a-level was adjusted with Bonferroni's correction. 


\section{Extended Data}

a -CD4-Cre $(\mathrm{n}=5)$

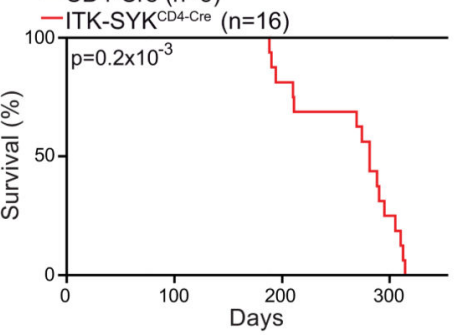

b

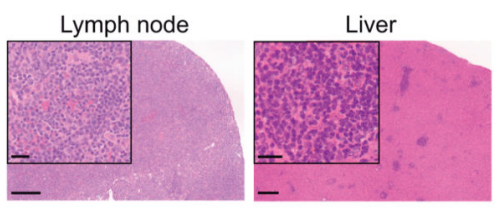

c $\square$ Control Geontrol
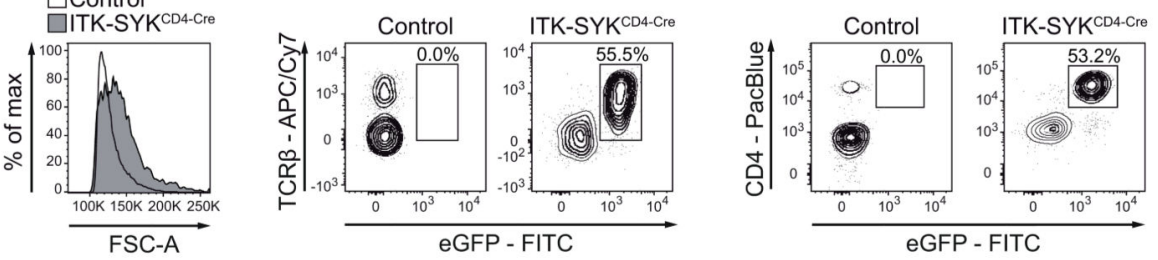

d

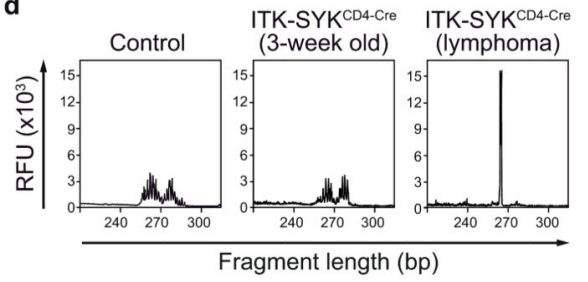

e Recipients f -ITK-SYK $K^{\mathrm{CD} 4-C r e}$ polyclonal (3-week old, $n=4$ )
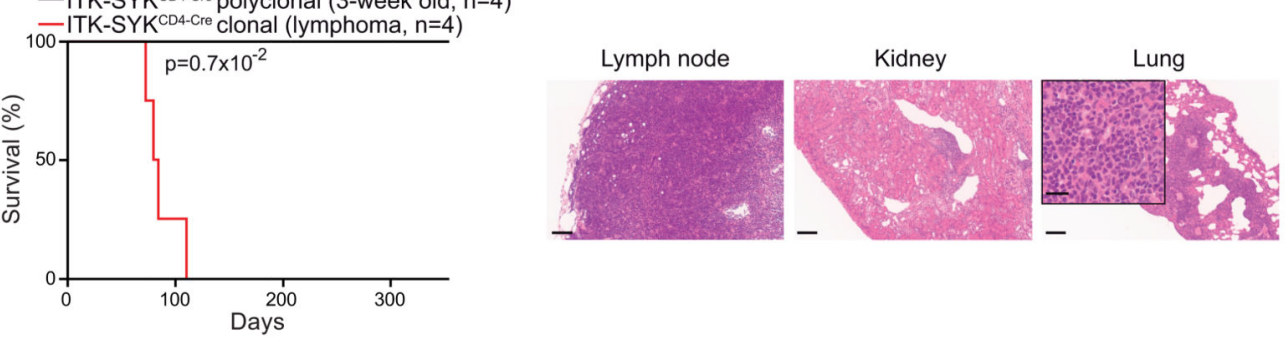

g $\square$ Control
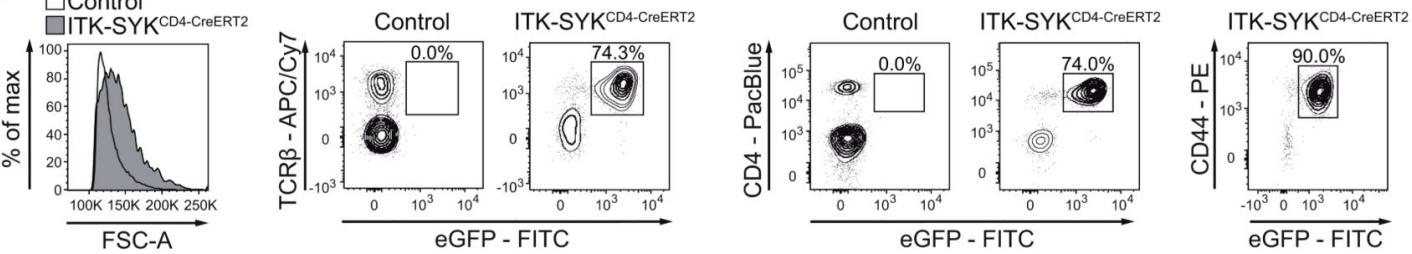

Extended Data Figure 1. Phenotypic characterization of ITK-SYK-induced lymphomas. a, Survival curves for the ITK-SYK ${ }^{\mathrm{CD} 4-C r e}$ mice and littermate controls (CD4-Cre). The pvalue was determined using the two-sided log-rank test, and the median survival was 281 days vs. not reached.

b, Histologies of lymph node and liver after H\&E staining of tissues from a sick ITKSYK ${ }^{\mathrm{CD} 4-\mathrm{Cre}}$ mouse on a C57BL/6 genetic background. The architecture of the lymph node 
is disrupted by a diffuse infiltration of lymphoblastoid cells. Multinodal perivascular infiltrations of lymphoblasts are also detectable in the liver. Scale bars represent $200 \mu \mathrm{m}$ and $20 \mu \mathrm{m}$ (insets).

c, Flow cytometric analyses of forward scatter area (FSC-A), which was used as a parameter to detect cell size, and TCR $\beta$, CD4 and eGFP expression in lymphoma tissues from a sick ITK-SYK ${ }^{\mathrm{CD} 4-C r e}$ mouse. Peripheral lymphocytes from a CD4-Cre littermate mouse served as the control.

d, GeneScan analysis of TCR gene rearrangements. Fragment size distributions of fluorochrome-labelled PCR products of the $\mathrm{V} \beta 1-20 ; \mathrm{J} \beta 2$ junction demonstrating polyclonal repertoires of a healthy CD4-Cre mouse (Control), a 3-week-old ITK-SYK ${ }^{\mathrm{CD} 4-C r e}$ mouse and clonality of the ITK-SYK ${ }^{\mathrm{CD}} 4$-Cre lymphoma sample. RFU, relative fluorescence unit e, Survival curves for C57BL/6 mice ( $\mathrm{n}=4$ recipients for each donor group) which had received polyclonal ITK-SYK ${ }^{\mathrm{CD}} 4$-Cre cells $\left(3 \times 10^{6}\right)$ from 3-week old ITK-SYK ${ }^{\mathrm{CD} 4-\mathrm{Cre}}$ mice or clonal ITK-SYK ${ }^{\mathrm{CD} 4-\mathrm{Cre}}$ cells from diseased ITK-SYK ${ }^{\mathrm{CD} 4-\mathrm{Cre}}$ mice with lymphoma ( $\mathrm{n}=4$ donors for each group). $\mathrm{P}=$ two-sided log-rank test

f, Histological analysis of lymphoma samples in tamoxifen-induced ITK-SYK ${ }^{C D} 4-C r e E R T 2$ animals. Lymph node with a loss of histomorphological architecture. Multifocal nodular infiltrations of lymphoblasts into kidney and lung are shown. Scale bars represent $100 \mu \mathrm{m}$ and $20 \mu \mathrm{m}$ (inset).

g, Flow cytometric analyses of TCR $\beta$-, CD4- and CD44-stained splenic cells in a tamoxifeninduced ITK-SYK ${ }^{\mathrm{CD} 4-C r e E R T 2}$ mouse and wild-type littermate (Control).

$\mathbf{b}, \mathbf{c}, \mathbf{d}, \mathbf{f}, \mathbf{g}$, Representative data from at least ten analysed mice. 
a

-Rosa26 $6^{\text {LSL-PB;ATP2;CD4-Cre }(n=5)}$

-Rosa26 $6^{\text {LSL-ITK-SYK;CD4-Cre }(n=14)}$

-Rosa26 ${ }^{\text {LSL-ITK-SYK;Rosa26 }}{ }^{\text {LSL-PB;ATP2;CD4-Cre }(n=30)}$

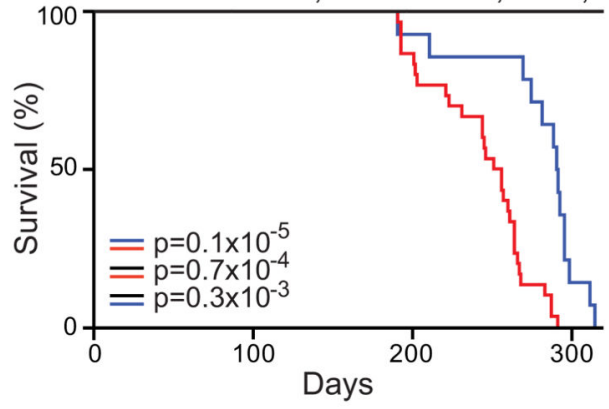

C

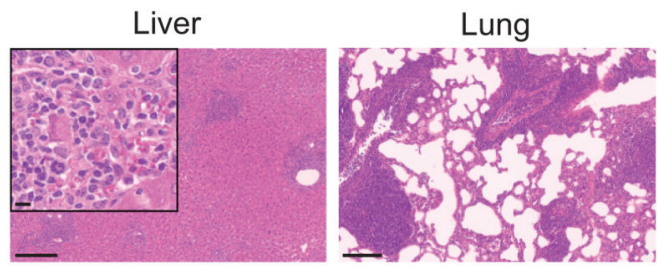

b

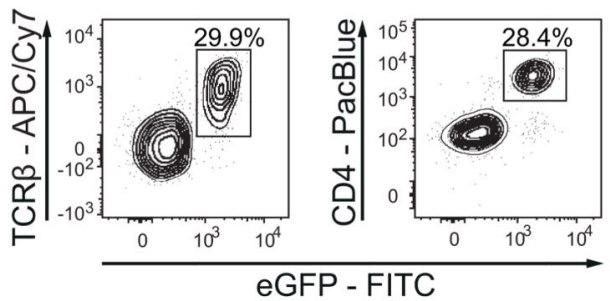

d

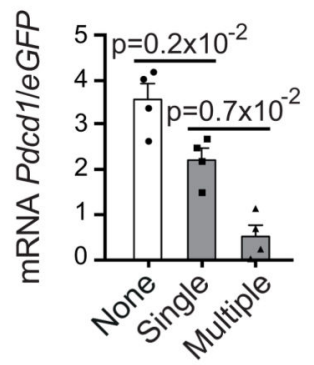

Pdcd1 insertions

Transcript: Pdcd1-001

ENSMUST00000027507.7

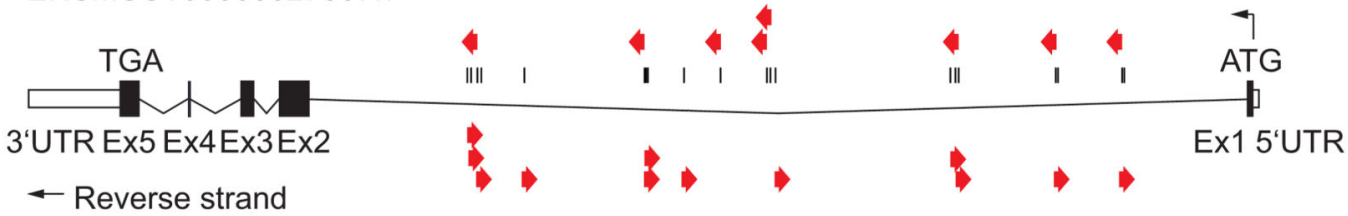

$\leftarrow$ Reverse strand

Antisense orientation:

Ex1f

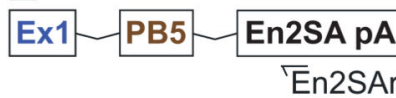

Pdcd1 Ex1

PB5

En2SA

Sense orientation:

LunSDf

MSCV LunSD $\frac{\text { Ex2 }}{\bar{E} \times 2 r}$

5"...AGGATCTCTCAGAGGTCCCCAATGGGC...3،

Extended Data Figure 2. Lymphomas with transposon insertions within Pdcd1.

a, Overall survival of the Rosa26 $6^{\text {LSL-ITK-SYK;Rosa26 }}$ LSL-PB;ATP2;CD4-Cre mice (red) and

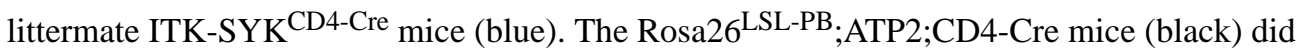
not display any signs of disease. The p-value was determined using two-sided log-rank test, and the median survival was 255 days vs. 289 days.

b, Flow cytometric analyses of TCR $\beta$, CD4 and eGFP expression in a typical lymphoma sample from a Rosa26 ${ }^{\text {LSL-ITK-SYK;Rosa26 }}{ }^{\text {LSL-PB;ATP2;CD4-Cre mouse. }}$ 
c, Histological analysis of the mouse shown in $\mathbf{b}$ with multinodal perivascular infiltrations of lymphoblasts into the liver (left) and lung (right). Scale bars represent $200 \mu \mathrm{m}$ and $20 \mu \mathrm{m}$ (inset).

d, Pdcd1 mRNA transcript levels in eGFP ${ }^{+}$FACS-sorted peripheral lymphocytes from Rosa26 $6^{\text {LSL-ITK-SYK }}$;Rosa26 ${ }^{\text {LSL-PB; }}$;ATP2;CD4-Cre lymphoma samples without any detectable transposon cassettes within the Pdcd1 locus (None, $\mathrm{n}=4$ ) or with a single insertion (Single, $\mathrm{n}=4$ ) or multiple Pdcd1-located transposon cassettes (Multiple, $\mathrm{n}=4$ ). Pdcd1 expression levels were normalized to eGFP transcript levels. $\mathrm{P}=$ Tukey's post hoc test. Shown are the mean \pm s.e.m and individual data points.

e, Twenty unique transposon integration sites mapped to the murine Pdcd1 locus. The vertical black bars indicate the genomic positions of the insertion. The red arrows indicate the orientations of the transposon cassettes.

f, Nucleotide sequences of the Pdcd1-piggyBac mRNA splice junctions as determined by Sanger sequencing of amplified cDNA from Rosa26 $6^{\text {LSL-ITK-SYK;Rosa26 }} 6^{\text {LSL-PB;ATP2;CD4- }}$ Cre lymphoma samples. The cDNA was generated from sorted $\mathrm{eGFP}^{+}$lymphoma cells in which at least one Pdcd1 transposon cassette could be detected. Note that the 5' piggyBac transposon-specific inverted terminal repeat nucleotide sequence (PB5) can function as a cryptic splice acceptor and a splice donor site. Hence, PB5 may act as a gene trapping sequence by itself ${ }^{14}$. En2SA, exon 2 located murine Engrailed 2 splice acceptor; pA, SV40 polyadenylation signal; MSCV, murine stem cell virus long terminal repeat; LunSD, splice donor from exon 1 of murine Foxf2.

b, c, Representative data from at least ten analysed mice. $\mathbf{f}$, The splice junctions were amplified from the cDNA of at least eight lymphomas. 
a

Human chromosome 2

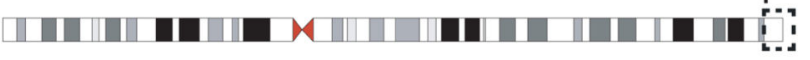

HES6 HDAC4 GPC1 PASK PDCD1

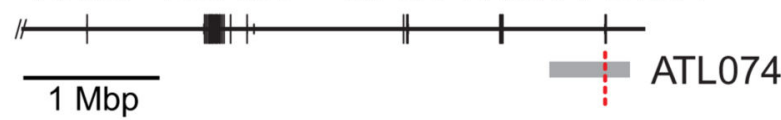

Copy Number Gain

PDCD1 locus

RNAseq PBMCs from healthy donor

$\square$ RNAseq ATL074

$\leftarrow$ Reverse strand

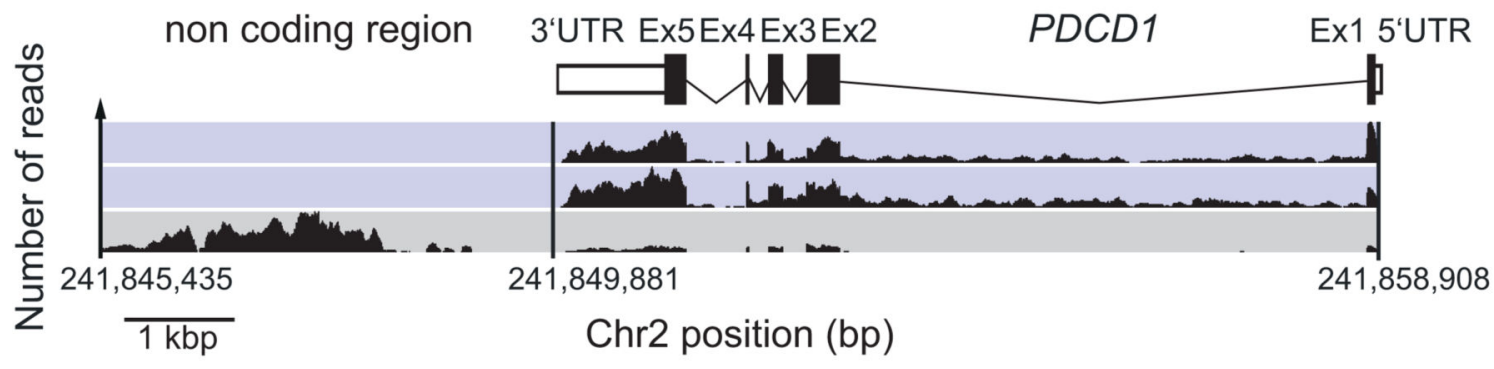

b

Human chromosome 2 $2 q 37.3$

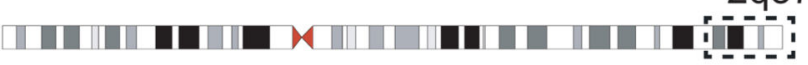
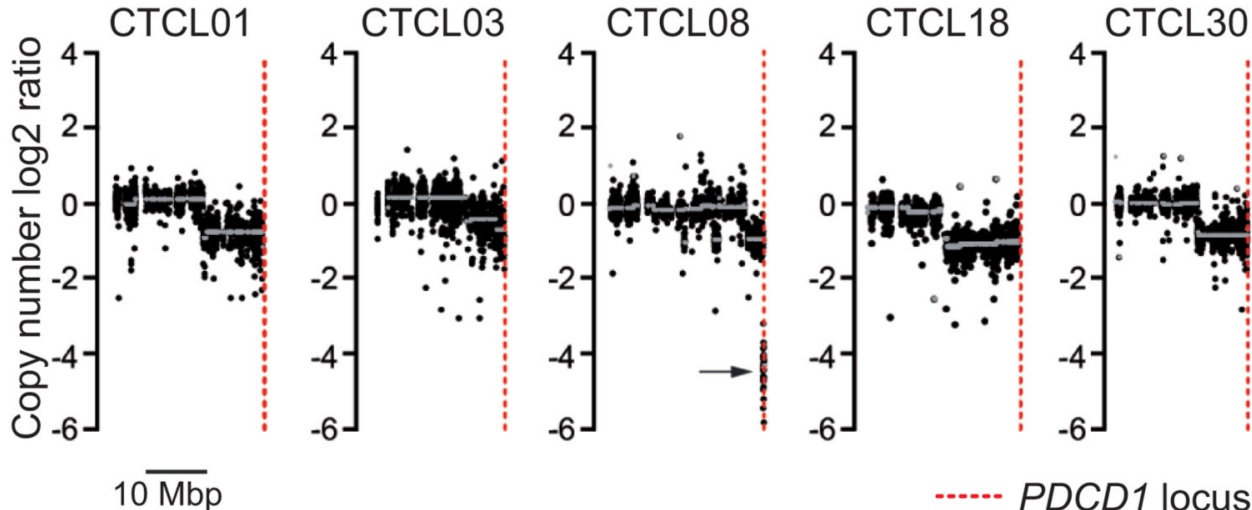

$10 \mathrm{Mbp}$

PDCD1 locus

Extended Data Figure 3. PDCD1 alterations in human lymphomas.

a, The dashed box indicates the genomic region q37.3 on human chromosome 2 that is shown in more detail in the middle of the panel (top). The grey horizontal bar indicates the genomic region that was affected by a PDCD1 copy number gain in T-NHL patient ATL0742 (middle). Histograms showing the number of human genome-aligned RNAseq reads from peripheral blood mononuclear cells (PBMCs) of two healthy donors and lymphoma cells of patient ATL074 at the PDCD1 locus and a non-coding region near the PDCD1 3' UTR (bottom). 
b, The dashed box indicates the genomic region on human chromosome 2 that is shown in detail in the lower part of the panel (top). CNAs in PDCD1 that were detected in CTCL patients6 are shown (bottom). The vertical dashed lines indicate the genomic position of the PDCD1 locus. Black dots represent the logarithmic tumour/normal copy number ratio; each dot represents $1 \mathrm{kbp}$. The grey lines indicate logarithmic value of the median tumour/normal copy number ratio. The arrow indicates a bi-allelic PDCD1 loss in sample CTCL08.

CNA, copy number aberration; kbp, kilo base pairs; Mbp, million base pairs

a

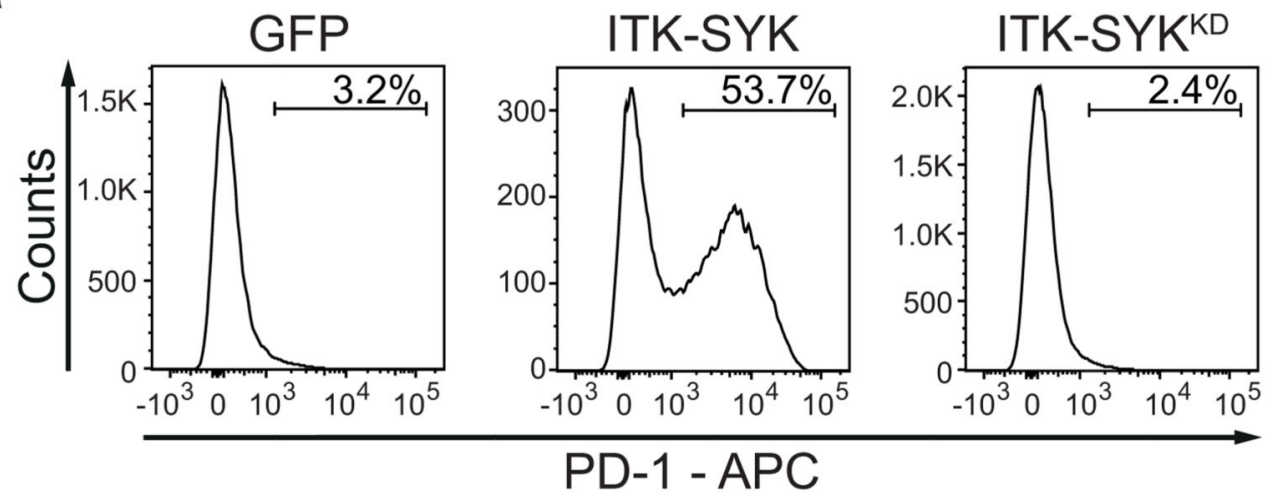

b

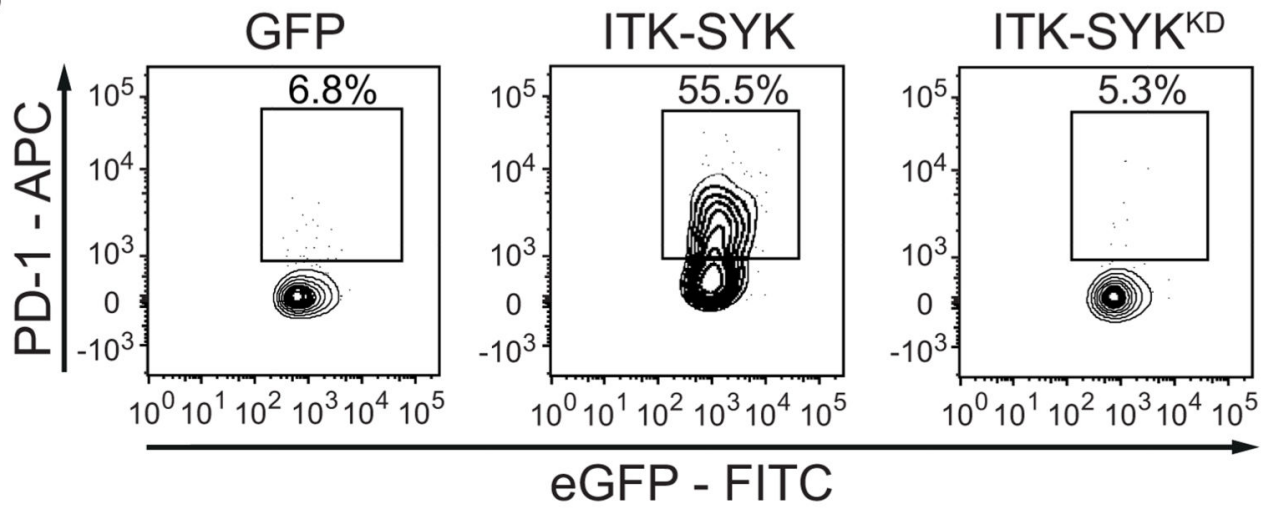

C
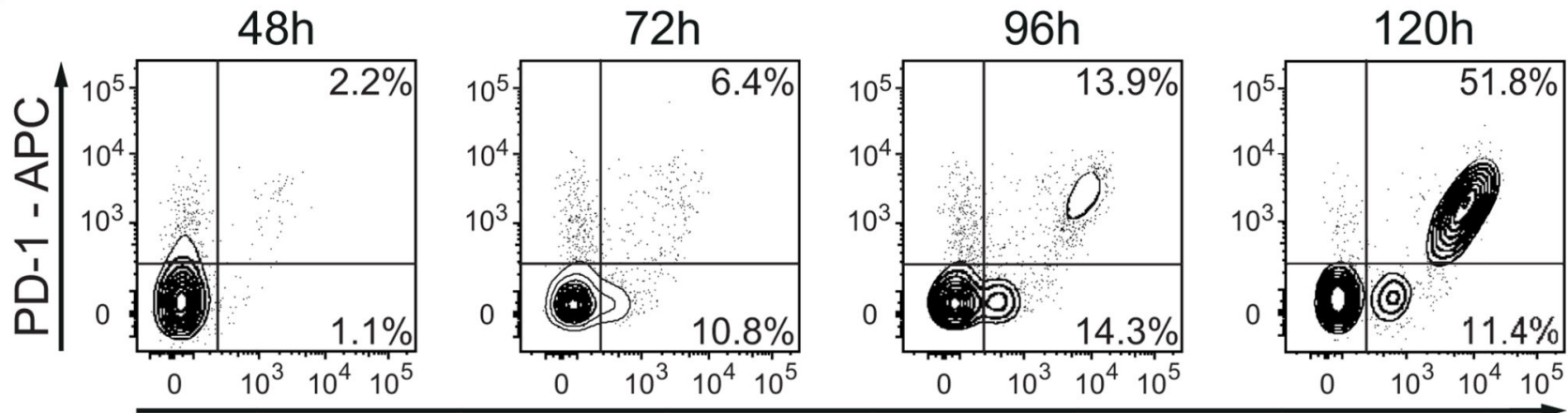

eGFP - FITC

Extended Data Figure 4. Oncogenic T cell signalling induces a PD-1 inhibitory loop. 
$\mathbf{a}, \mathbf{b}$, Jurkat $\mathrm{T}$ cells $(\mathbf{a})$ or primary human $\mathrm{CD} 4^{+} \mathrm{T}$ cells $(\mathbf{b})$ were infected with retroviruses carrying ITK-SYK or kinase-defective ITK-SYK ${ }^{\mathrm{KD}}$ together with GFP or GFP alone (control; GFP). PD-1 surface expression was determined by flow cytometry.

c, PD-1 expression after acute ITK-SYK signalling in primary T cells from tamoxifen-

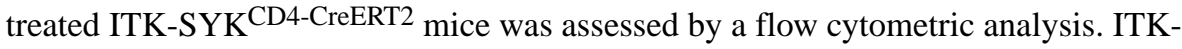
SYK $^{\mathrm{CD} 4-C r e E R T 2}$ mice received $1 \mathrm{mg}$ tamoxifen, and the cells were harvested 48 hours later, cultured in vitro and analysed at the indicated time points.

a, b The data from three experiments with similar results. $\mathbf{c}$ Representative results from three analysed mice.

a
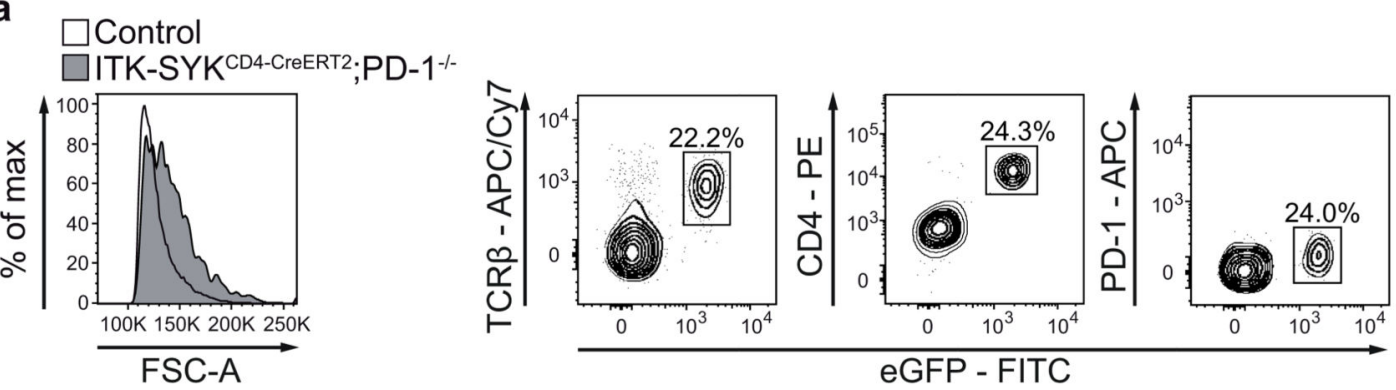

b Kidney
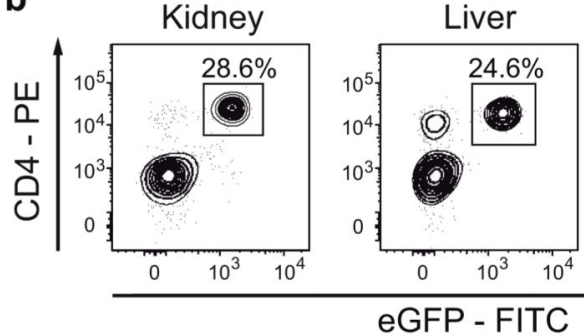

d
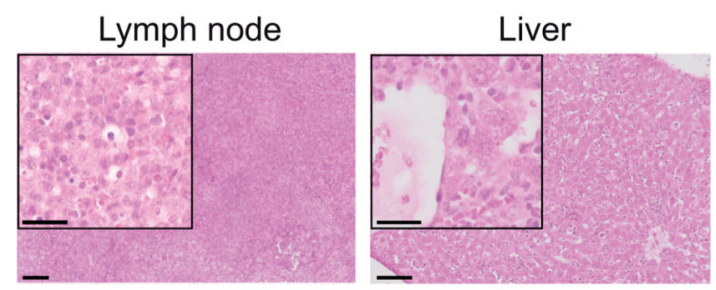

f

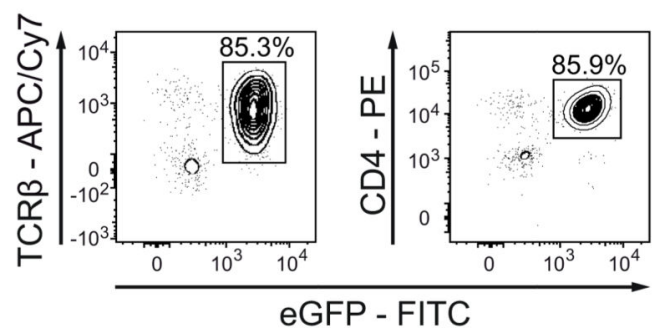

g
Lymph node
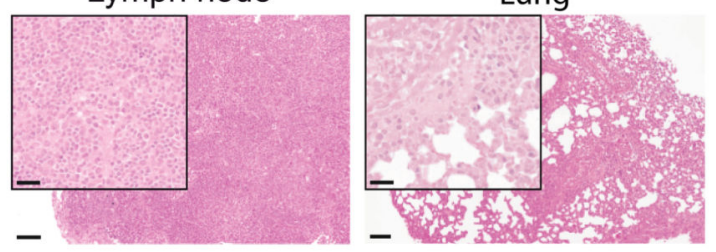

e $\square$ Control

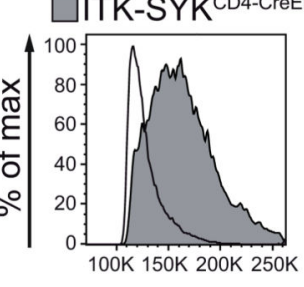

FSC-A

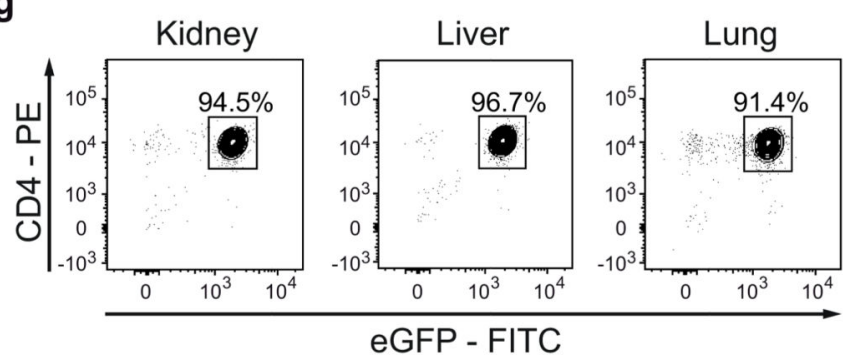

Extended Data Figure 5. Phenotypic characterization of PD-1 deficient lymphomas. 
a, b, Flow cytometric analyses of TCR $\beta$, CD4, PD-1 and eGFP expression in single-cell suspensions from the spleen (a) or kidney, liver and lung (b) from an ITK$\mathrm{SYK}^{\mathrm{CD} 4-\mathrm{CreERT} 2}$;PD-1 $1^{-/-}$mouse at one week after tamoxifen (TAM) injection. Flow cytometric analysis of forward scatter area (FSC-A) was used as a parameter to detect cell size.

c, Lymph node and lung histology by H\&E staining of tissues from a diseased ITK$\mathrm{SYK}^{\mathrm{CD} 4-\mathrm{CreERT} 2}$;PD-1 ${ }^{-/-}$mouse one week after tamoxifen induction. The lymph node architecture was disrupted by a diffuse neoplastic lymphoid infiltration. In the lung, neoplastic lymphoid cells were located in intra- and perivascular regions with a multifocal nodal infiltration pattern. Scale bars represent $100 \mu \mathrm{m}$ and $20 \mu \mathrm{m}$ (inset). Scale bars represent $100 \mu \mathrm{m}$ and $20 \mu \mathrm{m}$ (insets).

d, Histology by H\&E staining of lymph node and liver tissues of an NSG recipient mouse 13 days after the transplantation of $5 \times 10^{7}$ lymphomatous ITK-SYK ${ }^{\text {CD4-CreERT2 }}$ PD- $1^{-/-}$ splenocytes. Abnormal infiltration of lymphoblastoid cells is visible. Scale bars represent $100 \mu \mathrm{m}$ and $20 \mu \mathrm{m}$ (insets).

$\mathbf{e}, \mathbf{f}, \mathbf{g}$ Flow cytometric analysis of FSC-A and TCR $\beta$, CD4 and eGPF expression in lymphoma cells in the spleen (e, f), kidney, liver and lung (g) from the same mouse as in $\mathbf{d}$. $\mathbf{a}, \mathbf{b}, \mathbf{c}$ Representative data from three independent experiments, each with three biological replicates. d, e, f, $\mathbf{g}$ Representative results from one of six NSG recipient mice from two independent experiments. 
a
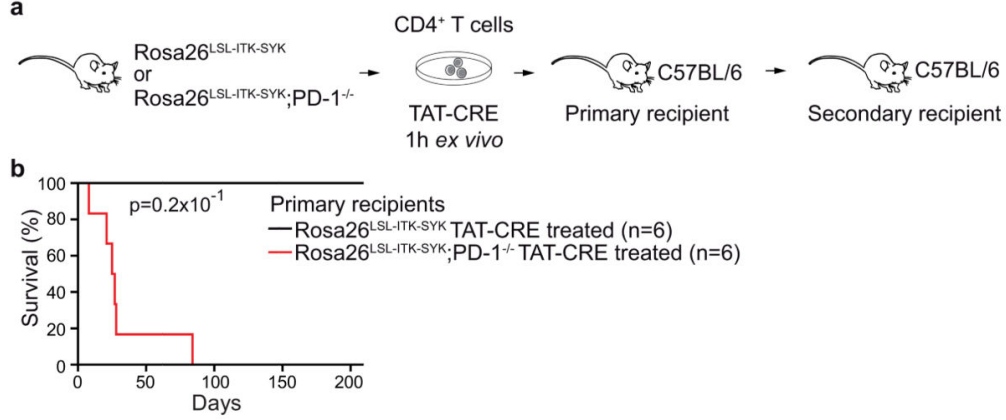

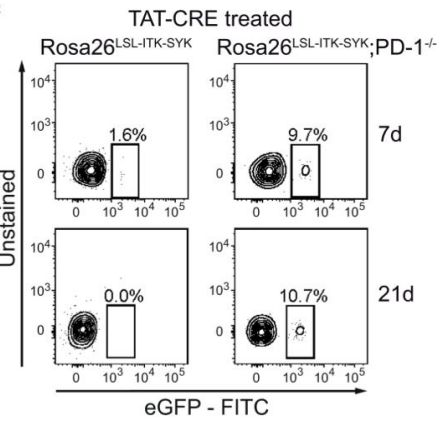

e $\square$ Control TAT-CRE treated $\square$ Rosa26 $6^{\text {LSLITK-SYK; }}$ PD- $1^{\%}$ TAT-CRE treated
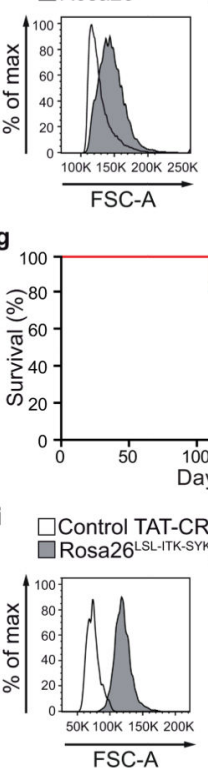

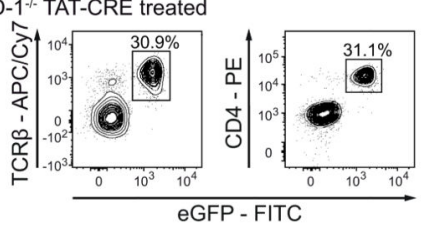

EGFP - FITC

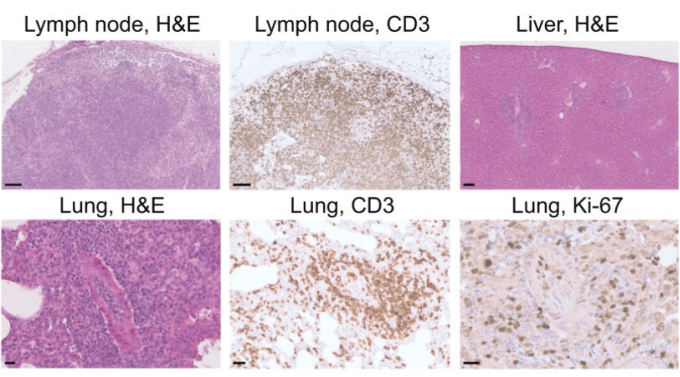

f

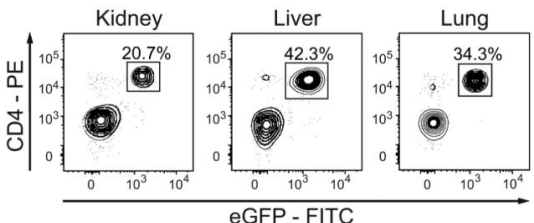

h

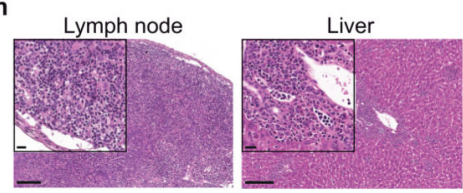

Extended Data Figure 6. Serial transplantation of PD-1-deficient lymphomas.

a, Representation of the experimental strategy for inducing ITK-SYK expression via TATCRE in PD-1-competent (Rosa26 $6^{\text {LSL-ITK-SYK }}$ ) or PD-1-deficient

(Rosa26 ${ }^{\text {LSL-ITK-SYK;PD-1 }}{ }^{-/}$) cells for subsequent transfer into wild-type C57BL/6 recipient mice.

b, Survival curves of the C57BL/6 recipients ( $\mathrm{n}=6$ recipients per genotype) transplanted with $5 \times 10^{5}$ TAT-CRE-treated Rosa26 $6^{\text {LSL-ITK-SYK }}$ or Rosa26 $6^{\text {LSL-ITK-SYK }}$ PD- $1^{-{ }^{-}} \mathrm{CD} 4^{+} \mathrm{T}$ cells ( $\mathrm{n}=6$ donor mice per genotype). $\mathrm{P}=$ two-sided log-rank test 
c, Flow cytometric analysis of ITK-SYK/eGFP expression in peripheral blood lymphocytes from C57BL/6 mice that received TAT-CRE-treated PD-1-competent (Rosa26 ${ }^{\text {LSL-ITK-SYK) }}$ or PD-1-deficient (Rosa26 ${ }^{\text {LSL-ITK-SYK;PD-1 }}{ }^{-{ }^{-}}$) cells on days 7 and 21 after transplantation. The genotypes of the donor mice are indicated.

d, Histology and immunohistochemistry of the indicated organs from a sick primary recipient mouse after the transfer of TAT-CRE-treated Rosa26 $6^{\mathrm{LSL}-\mathrm{ITK}-\mathrm{SYK}} ; \mathrm{PD}-1^{-/-} \mathrm{T}$ cells showing the expansion of a malignant T-lymphocyte population. Scale bars represent 100 $\mu \mathrm{m}$ (top row) and $20 \mu \mathrm{m}$ (bottom row).

$\mathbf{e}, \mathbf{f}$, Flow cytometric analysis of CD4-stained lymphocytes derived from the spleen (e) or indicated organs (f) of the mouse shown in d. FSC-A, forward scatter area, which was used as a parameter to detect cell size of splenic Rosa26 ${ }^{\mathrm{LSL}-I T K-S Y K} ; \mathrm{PD}-1^{-/-} \mathrm{T}$ cells compared to the TAT-CRE treated C57BL/6 CD4+ $\mathrm{T}$ cells (Control).

g, Survival of secondary C57BL/6 recipient mice $(n=6)$ that received $1 \times 10^{5}$ TAT-CREinduced Rosa26 $6^{\text {LSL-ITK-SYK;PD-1 }}{ }^{-/-}$lymphoma T-cells from diseased primary C57BL/6 recipients ( $\mathrm{n}=6$ biological replicates).

h, H\&E-stained histologic sections from lymph node and liver tissues from a diseased C57BL/6 mouse that had received $1 \times 10^{5}$ TAT-CRE-treated Rosa26 $6^{\text {LSL-ITK-SYK;PD- } 1^{-/-} \mathrm{T}}$ cells as the secondary recipient from a sick C57BL/6 primary recipient. Scale bars represent $100 \mu \mathrm{m}$ and $20 \mu \mathrm{m}$ (insets).

$\mathbf{i}, \mathbf{j}$, Flow cytometric analysis as in $\mathbf{e}, \mathbf{f}$ but for the secondary recipient from $\mathbf{h}$. c, Characteristic flow cytometric profiles measured in two independent experiments. $\mathbf{d}, \mathbf{e}, \mathbf{f}, \mathbf{h}, \mathbf{i}, \mathbf{j}$, Representative results from one out of six analysed mice. 


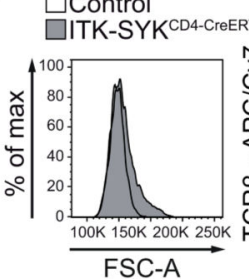

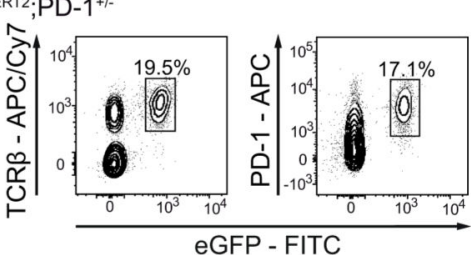

b

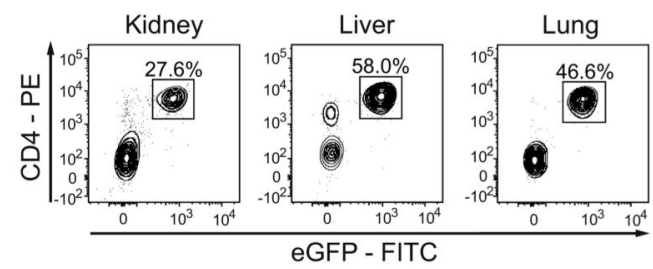

c

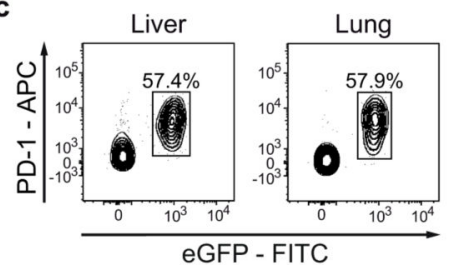

d
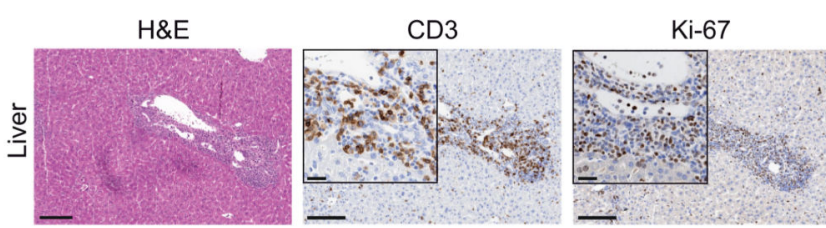

e

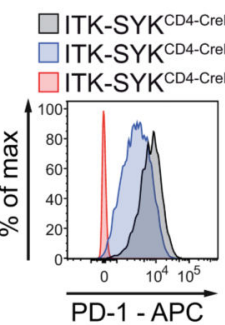

g
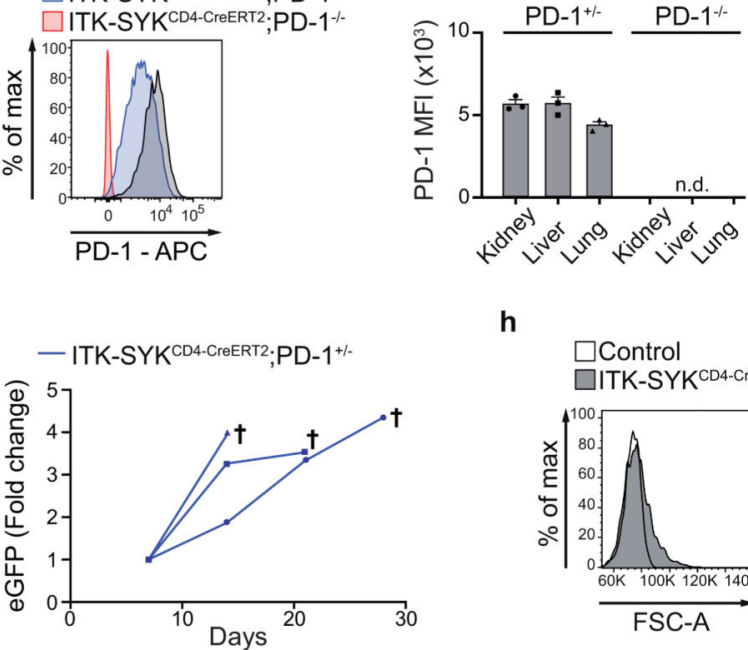

h

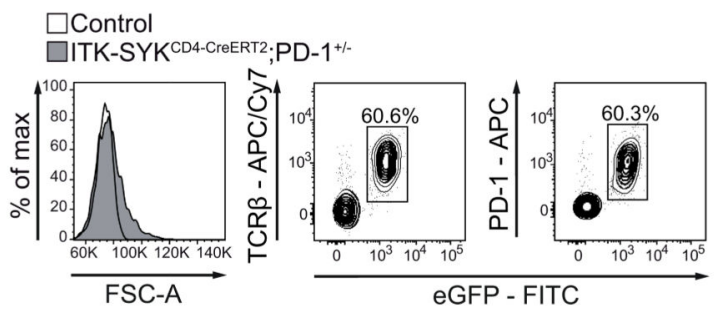

i

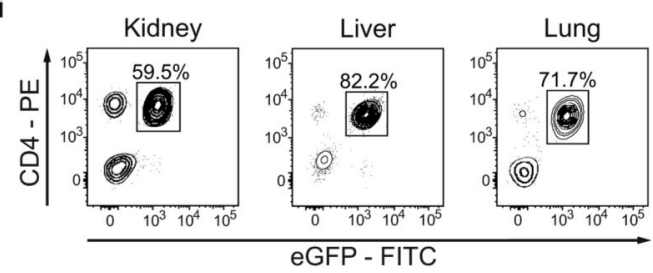

j
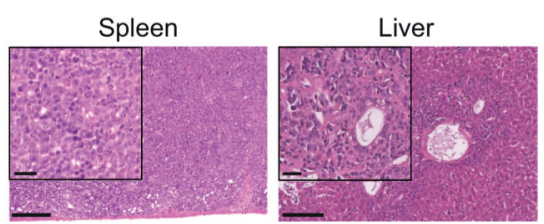

Extended Data Figure 7. Characterization of Pdcd1- heterozygous lymphomas.

a, Flow cytometric analysis of forward scatter area (FSC-A), which was used as a parameter to detect cell size, and TCR $\beta$, PD-1 and eGPF expression in splenic lymphoma cells from a diseased ITK-SYK ${ }^{\mathrm{CD} 4-C r e E R T 2}$;PD-1 ${ }^{+/-}$mouse at two weeks after tamoxifen injection (0.25 $\mathrm{mg}$ ). CD4 ${ }^{+} \mathrm{T}$ cells from an untreated wild-type C57BL/6 mouse (Control) served as control. b, c, Flow cytometric analysis of CD4, eGFP and PD-1 expression in single-cell suspensions of the indicated dissociated organs from the same mouse as in a. 
d, Histology after H\&E staining and immunohistochemical staining with anti-CD3 or antiKi-67 antibody of the liver tissue from the same animal as in $\mathbf{a}, \mathbf{b}, \mathbf{c}$. Abnormal infiltration of lymphoblastoid cells is visible. Scale bars represent $100 \mu \mathrm{m}$ and $20 \mu \mathrm{m}$ (insets).

e, Flow cytometric analyses of PD-1 receptor expression on ITK-SYK ${ }^{+} / \mathrm{GFP}^{+} \mathrm{T}$ cells from ITK-SYK $^{\text {CD4-CreERT2 }}$, ITK-SYK ${ }^{\text {CD4-CreERT2;PD- }}{ }^{+/-}$and ITK-SYK ${ }^{\text {CD4-CreERT2;PD- }}{ }^{-/-}$ mice are shown.

f, Flow cytometric analysis of PD-1 expression on ITK-SYK ${ }^{+} / \mathrm{GFP}^{+} \mathrm{T}$ lymphocytes. Cells isolated from the kidney, liver and lung of diseased ITK-SYK ${ }^{\mathrm{CD} 4-\mathrm{CreERT}} ; \mathrm{PD}-1^{+/-}$(labelled with PD-1 ${ }^{+/-}$) or ITK-SYK ${ }^{\mathrm{CD} 4-\mathrm{CreERT}}$;PD-1/- $\left(\right.$ labelled with PD-1 ${ }^{-/}$) mice from the experiment presented in Fig. 4c. Shown are the mean \pm s.d. and individual data points; n.d., not detectable.

g, Fifty million splenic cells from diseased ITK-SYK ${ }^{\mathrm{CD} 4-\mathrm{CreERT} 2}$;PD- ${ }^{+/-}$mice ( $\mathrm{n}=3$ donors) were intravenously transferred to NSG mice ( $n=3$ recipients). The fold changes of ITKSYK-expressing eGFP ${ }^{+}$lymphocytes in the peripheral blood of the recipients are indicated. $\dagger$ indicates animals that had to be euthanized because of lymphomas.

$\mathbf{h}, \mathbf{i}$, Flow cytometric analysis of the FSC-A, TCR $\beta$, PD-1, CD4 and eGFP expression in spleen cell suspension (h) or lymphoma cells isolated from the lung, kidney and liver (i) of an NSG mouse that received $5 \times 10^{7}$ splenic cells from a TAM-induced ITK$\mathrm{SYK}^{\mathrm{CD} 4-\mathrm{CreERT}}$;PD-1/- mouse. $\mathrm{CD} 4^{+}{ }^{+} \mathrm{T}$ cells from an untreated wild-type C57BL/6 mouse served as control.

$\mathbf{j}$, Spleen and liver histology after H\&E staining of organ sections of the NSG recipient mouse presented in $\mathbf{h}$, $\mathbf{i}$. Infiltration of a lymphoblastoid cell population in the spleen resulting in a complete loss of the normal architecture. Mixed periportal and intrasinusoidal infiltrations of neutrophilic granulocytes, lymphocytes and lymphoblasts into the liver with multifocal periportal hepatocellular necroses. Scale bars represent $100 \mu \mathrm{m}$ and $20 \mu \mathrm{m}$ (insets).

a, b, c, d, Representative data from three independent experiments, each with at least three biological replicates. e, Histograms representing one out of three analysed mice per genotype. f, PD-1 expression on $\mathrm{eGFP}^{+} \mathrm{T}$ cells isolated from the lung, kidney, and liver was measured in three biological replicates per genotype. $\mathbf{g}$, The data from a single experiment that was independently repeated once with similar results. $\mathbf{h}, \mathbf{i}$, Representative results from one of six analysed mice from two independent experiments. $\mathbf{j}$, The results are characteristic for three mice that were analysed in two independent experiments. 
a
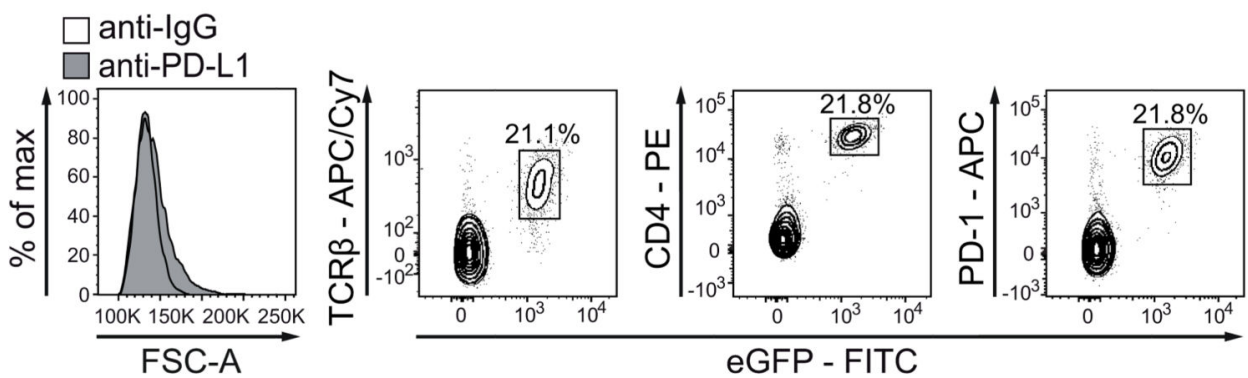

b
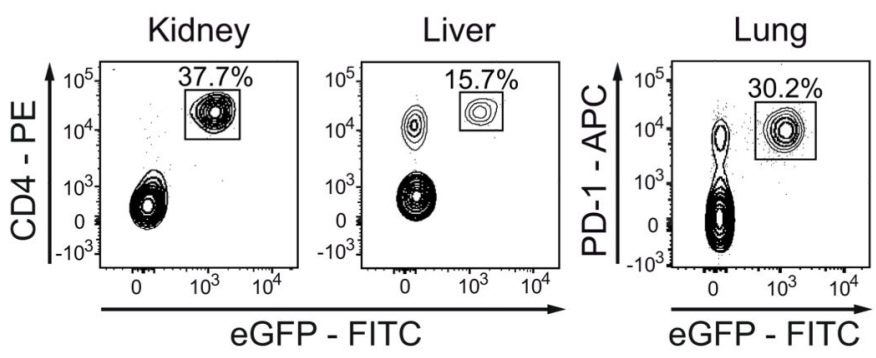

C
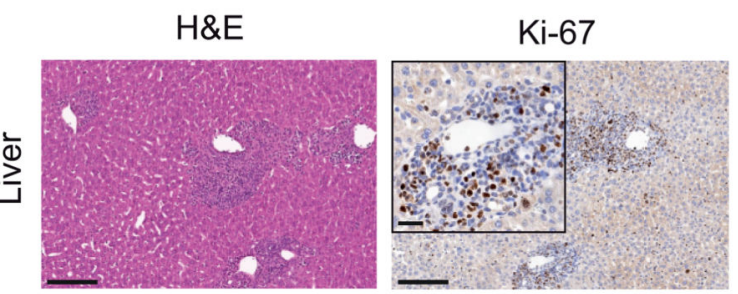

d

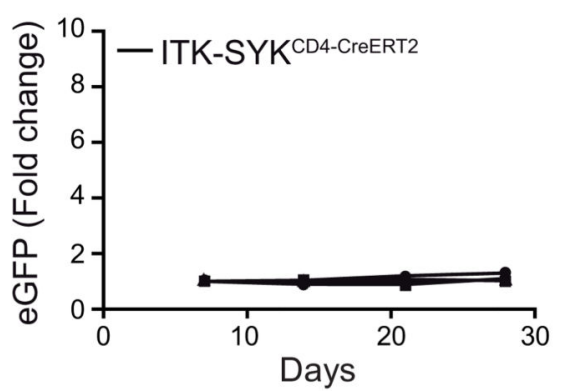

e

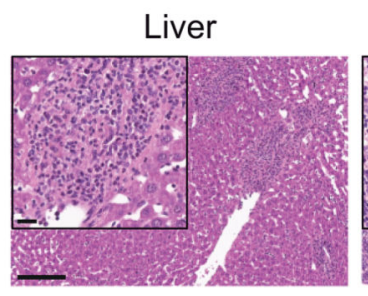

Lymph node

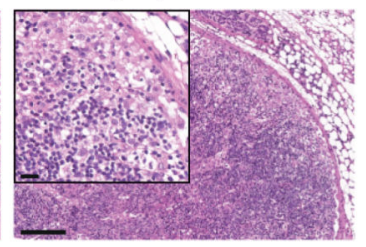

Extended Data Figure 8. Anti-PD-L1 triggers lethal ITK-SYK ${ }^{+}$lymphoproliferation.

$\mathbf{a}, \mathbf{b}, \mathbf{c}$, Analyses of the antibody-treated mice from the experiment shown in Fig. 4d. Flow cytometric data of forward scatter area (FSC-A), which was used as a parameter to detect cell size, and TCR $\beta$, CD4, PD-1 and eGPF expression in lymphomatous cells isolated from the spleen (a), kidney, liver and lung (b).

c, Histology and immunohistochemistry of liver sections from the same mouse demonstrating the expansion of blastoid T-cells. Scale bars represent $100 \mu \mathrm{m}$ and $20 \mu \mathrm{m}$ (inset).

d, Fifty million splenic cells from anti-PD-L1-treated, sick ITK-SYK ${ }^{\text {CD4-CreERT2 }}$ mice ( $\mathrm{n}=3$ biological replicates) were intravenously transferred to NSG recipient mice $(n=3)$. The fold change of $\mathrm{eGFP}^{+}$lymphocytes in the peripheral blood of recipients over time is shown.

e, Liver and lymph node histology after H\&E staining of tissue sections from a diseased

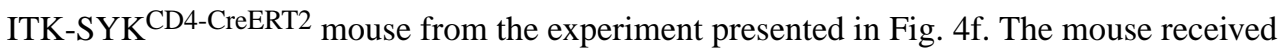
an anti-PD-1 antibody treatment that started ten days after tamoxifen administration.

a, b, c, Representative data from one out of nine analysed mice in four independent experiments. d, The data from a single experiment that was repeated once with similar results. e, Histologies are characteristic for four mice that were analysed in two independent experiments. 
a
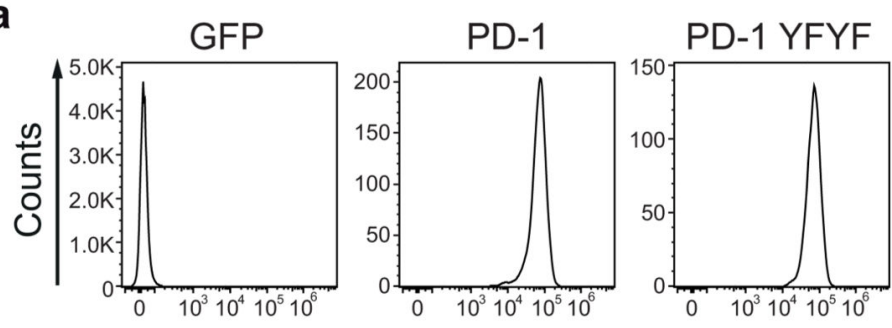

PD-1 - APC

b

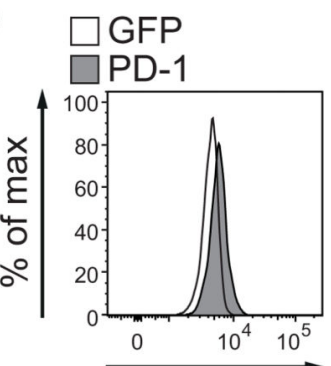

PTEN - PE
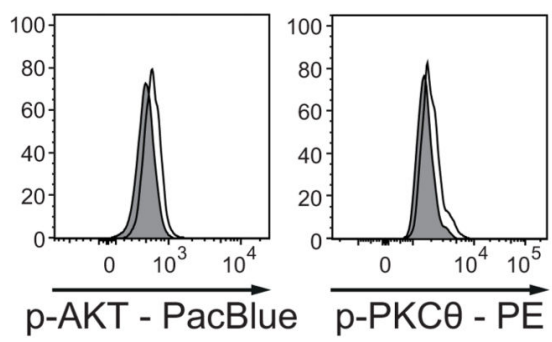

C
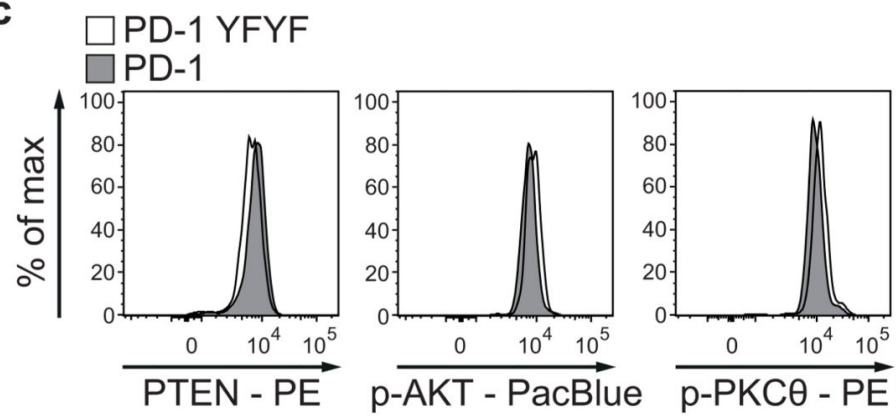

d

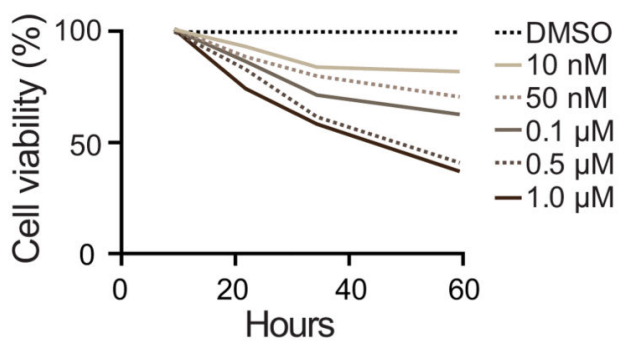

e $\square$ Vehicle

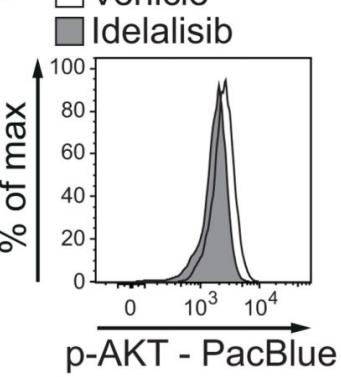

Extended Data Figure 9. PD-1 regulates PI3K signalling.

a, Human T-NHL HuT 78 cells were infected with retroviruses carrying wild-type PD-1 (PD-1) or a signalling-incompetent variant of PD-1 (PD-1 YFYF; ITIM/ITSM motifs mutated in the PD-1 cytoplasmic tail) together with GFP or GFP alone (control; GFP). PD-1 surface expression was determined by flow cytometry.

b, Intracellular flow cytometric analyses of PTEN, p-AKT and p-PKC $\theta$ levels in wild-type PD-1- (PD-1) or GFP only-transduced (GFP) HuT 78 cells that had been co-cultured with PD-L1-expressing human dendritic cells for 24 hours. 
c, Intracellular flow cytometric analyses from a similar experiment as in $\mathbf{b}$ but with wildtype-PD-1 (PD-1) and Y223F/Y248F-mutated-PD-1 (PD-1 YFYF) transduced HuT 78 cells. d, TAT-CRE-induced Rosa26 LSL-ITK-SYK;PD-1 ${ }^{-/-}$cells isolated from diseased C57BL/6 recipient mice (see Extended Data Fig. 6b) were cultured in vitro in the presence of the indicated concentration of the PI3K $\delta$ inhibitor idelalisib or DMSO. Cell viability was determined over time.

e, Ex vivo phosphorylation status of the AKT kinase after the oral administration of idelalisib $(10 \mathrm{mg} / \mathrm{kg})$ or vehicle into mice. ITK-SYK ${ }^{\mathrm{CD} 4-\mathrm{CreERT} 2}$;PD-1 ${ }^{-/-} \mathrm{T}$ cells were induced in vivo with tamoxifen $(0.25 \mathrm{mg})$. On day five after induction, $5 \times 10^{4} \mathrm{eGFP}^{+} \mathrm{T}$ cells were transplanted into NSG recipient mice. Five days later, the mice received a single gavage of idelalisib $(10 \mathrm{mg} / \mathrm{kg})$ or vehicle control. Four hours later, spleen-derived singlecell suspensions were analysed by flow cytometry via Phosflow.

a, Results from two independent experiments with comparable outcomes. b, $\mathbf{c}$ Experiments were performed three times, each with similar results. d, Experiment was performed with four biological replicates; one representative replicate is shown. e, Experiment was performed twice with similar results. 


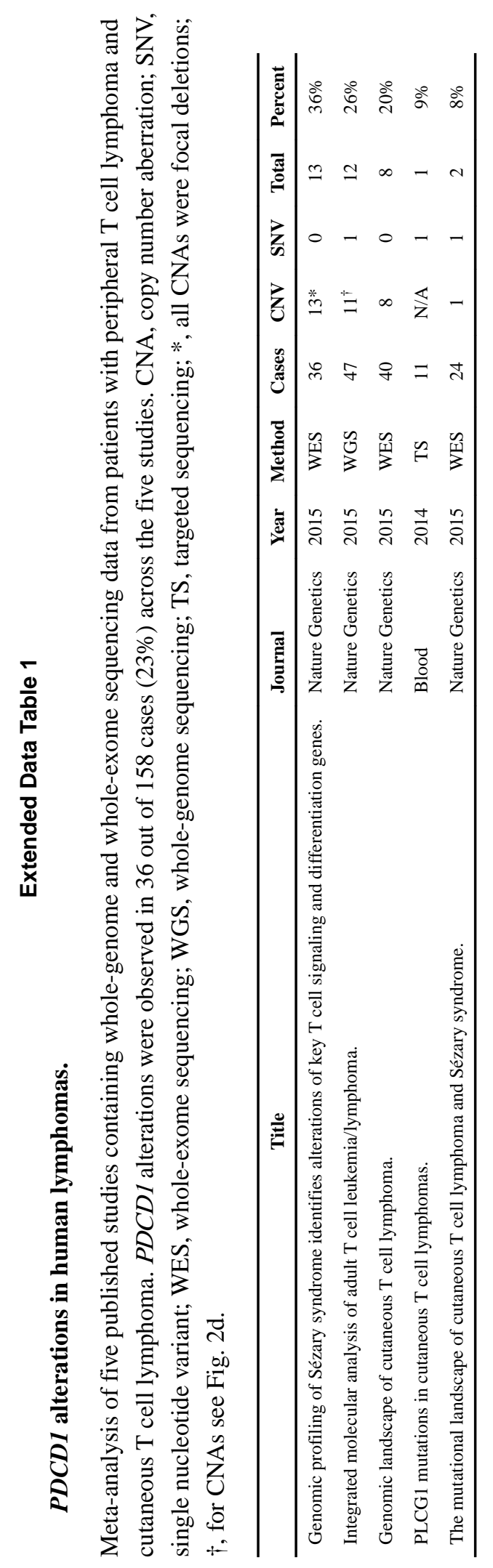

Nature. Author manuscript; available in PMC 2018 May 15. 


\section{Supplementary Material}

Refer to Web version on PubMed Central for supplementary material.

\section{Acknowledgements}

We thank Nicole Prause and Kerstin Burmeister for providing excellent technical assistance and S. Ogawa, K. Kataoka, R. P. Lifton and J. Choi for providing access to NGS data from the T cell lymphoma patients. This study used data generated by the Department of Pathology and Tumor Biology of Kyoto University. This work was supported by research grants from the DFG (SFB 1054/B01 and RU 695/6-1) and ERC (FP7, grant agreement No. 322865) awarded to J.R.

\section{References}

1. Casulo C, et al. T-Cell Lymphoma: Recent Advances in Characterization and New Opportunities for Treatment. Journal of the National Cancer Institute. 2017; 109doi: 10.1093/jnci/djw248

2. Kataoka K, et al. Integrated molecular analysis of adult T cell leukemia/lymphoma. Nat Genet. 2015; 47:1304-1315. DOI: 10.1038/ng.3415 [PubMed: 26437031]

3. Wang L, et al. Genomic profiling of Sezary syndrome identifies alterations of key T cell signalling and differentiation genes. Nat Genet. 2015; 47:1426-1434. DOI: 10.1038/ng.3444 [PubMed: 26551670]

4. da Silva Almeida AC, et al. The mutational landscape of cutaneous T cell lymphoma and Sezary syndrome. Nat Genet. 2015; 47:1465-1470. DOI: 10.1038/ng.3442 [PubMed: 26551667]

5. Vaque JP, et al. PLCG1 mutations in cutaneous T-cell lymphomas. Blood. 2014; 123:2034-2043. DOI: 10.1182/blood-2013-05-504308 [PubMed: 24497536]

6. Choi J, et al. Genomic landscape of cutaneous T cell lymphoma. Nat Genet. 2015; 47:1011-1019. DOI: 10.1038/ng.3356 [PubMed: 26192916]

7. Streubel B, Vinatzer U, Willheim M, Raderer M, Chott A. Novel t $(5 ; 9)(q 33 ; q 22)$ fuses ITK to SYK in unspecified peripheral T-cell lymphoma. Leukemia. 2006; 20:313-318. DOI: 10.1038/sj.leu. 2404045 [PubMed: 16341044]

8. Pechloff K, et al. The fusion kinase ITK-SYK mimics a T cell receptor signal and drives oncogenesis in conditional mouse models of peripheral $\mathrm{T}$ cell lymphoma. The Journal of experimental medicine. 2010; 207:1031-1044. DOI: 10.1084/jem.20092042 [PubMed: 20439541]

9. Mulloy JC. Peripheral T cell lymphoma: new model + new insight. The Journal of experimental medicine. 2010; 207:911-913. DOI: 10.1084/jem.20100608 [PubMed: 20439538]

10. Sledzinska A, et al. TGF-beta signalling is required for CD4(+) T cell homeostasis but dispensable for regulatory T cell function. PLoS biology. 2013; 11:e1001674.doi: 10.1371/journal.pbio. 1001674 [PubMed: 24115907]

11. Ishida $\mathrm{S}$, et al. Role for E2F in control of both DNA replication and mitotic functions as revealed from DNA microarray analysis. Molecular and cellular biology. 2001; 21:4684-4699. DOI: 10.1128/MCB.21.14.4684-4699.2001 [PubMed: 11416145]

12. Liberzon A, et al. The Molecular Signatures Database (MSigDB) hallmark gene set collection. Cell systems. 2015; 1:417-425. DOI: 10.1016/j.cels.2015.12.004 [PubMed: 26771021]

13. Whitfield ML, et al. Identification of genes periodically expressed in the human cell cycle and their expression in tumours. Molecular biology of the cell. 2002; 13:1977-2000. DOI: 10.1091/mbc. 02-02-0030 [PubMed: 12058064]

14. Rad R, et al. PiggyBac transposon mutagenesis: a tool for cancer gene discovery in mice. Science. 2010; 330:1104-1107. DOI: 10.1126/science.1193004 [PubMed: 20947725]

15. Friedrich MJ, et al. Genome-wide transposon screening and quantitative insertion site sequencing for cancer gene discovery in mice. Nature protocols. 2017; 12:289-309. DOI: 10.1038/nprot. 2016.164 [PubMed: 28079877]

16. Rad R, et al. A conditional piggyBac transposition system for genetic screening in mice identifies oncogenic networks in pancreatic cancer. Nat Genet. 2015; 47:47-56. DOI: 10.1038/ng.3164 [PubMed: 25485836] 
17. Sarver AL, Erdman J, Starr T, Largaespada DA, Silverstein KA. TAPDANCE: an automated tool to identify and annotate transposon insertion CISs and associations between CISs from next generation sequence data. BMC Bioinformatics. 2012; 13:154.doi: 10.1186/1471-2105-13-154 [PubMed: 22748055]

18. Riley JL. PD-1 signalling in primary T cells. Immunological reviews. 2009; 229:114-125. DOI: 10.1111/j.1600-065X.2009.00767.x [PubMed: 19426218]

19. Francisco LM, Sage PT, Sharpe AH. The PD-1 pathway in tolerance and autoimmunity. Immunological reviews. 2010; 236:219-242. DOI: 10.1111/j.1600-065X.2010.00923.x [PubMed: 20636820]

20. Patsoukis N, Li L, Sari D, Petkova V, Boussiotis VA. PD-1 increases PTEN phosphatase activity while decreasing PTEN protein stability by inhibiting casein kinase 2. Molecular and cellular biology. 2013; 33:3091-3098. DOI: 10.1128/MCB.00319-13 [PubMed: 23732914]

21. Chemnitz JM, Parry RV, Nichols KE, June CH, Riley JL. SHP-1 and SHP-2 associate with immunoreceptor tyrosine-based switch motif of programmed death 1 upon primary human $\mathrm{T}$ cell stimulation, but only receptor ligation prevents T cell activation. J Immunol. 2004; 173:945-954. [PubMed: 15240681]

22. Sheppard KA, et al. PD-1 inhibits T-cell receptor induced phosphorylation of the ZAP70/CD3zeta signalosome and downstream signaling to PKCtheta. FEBS letters. 2004; 574:37-41. DOI: 10.1016/j.febslet.2004.07.083 [PubMed: 15358536]

23. Keir ME, Freeman GJ, Sharpe AH. PD-1 regulates self-reactive CD8+ T cell responses to antigen in lymph nodes and tissues. J Immunol. 2007; 179:5064-5070. [PubMed: 17911591]

24. Nolden L, et al. Site-specific recombination in human embryonic stem cells induced by cellpermeant Cre recombinase. Nat Methods. 2006; 3:461-467. DOI: 10.1038/nmeth884 [PubMed: 16721380]

25. Brahmer JR, et al. Safety and activity of anti-PD-L1 antibody in patients with advanced cancer. The New England journal of medicine. 2012; 366:2455-2465. DOI: 10.1056/NEJMoa1200694 [PubMed: 22658128]

26. Gopal AK, et al. PI3Kdelta inhibition by idelalisib in patients with relapsed indolent lymphoma. The New England journal of medicine. 2014; 370:1008-1018. DOI: 10.1056/NEJMoa1314583 [PubMed: 24450858]

27. Kulpa DA, et al. PD-1 coinhibitory signals: the link between pathogenesis and protection. Seminars in immunology. 2013; 25:219-227. DOI: 10.1016/j.smim.2013.02.002 [PubMed: 23548749]

28. Jordan B. [First use of CRISPR for gene therapy]. Medecine sciences : M/S. 2016; 32:1035-1037. DOI: 10.1051/medsci/20163211024 [PubMed: 28008849]

29. Lee PP, et al. A critical role for Dnmt1 and DNA methylation in T cell development, function, and survival. Immunity. 2001; 15:763-774. [PubMed: 11728338]

30. Kim D, Langmead B, Salzberg SL. HISAT: a fast spliced aligner with low memory requirements. Nat Methods. 2015; 12:357-360. DOI: 10.1038/nmeth.3317 [PubMed: 25751142]

31. Pertea M, et al. StringTie enables improved reconstruction of a transcriptome from RNA-seq reads. Nat Biotechnol. 2015; 33:290-295. DOI: 10.1038/nbt.3122 [PubMed: 25690850]

32. Frazee AC, et al. Ballgown bridges the gap between transcriptome assembly and expression analysis. Nat Biotechnol. 2015; 33:243-246. DOI: 10.1038/nbt.3172 [PubMed: 25748911]

33. Spinelli L, Carpentier S, Montanana Sanchis F, Dalod M, Vu Manh TP. BubbleGUM: automatic extraction of phenotype molecular signatures and comprehensive visualization of multiple Gene Set Enrichment Analyses. BMC Genomics. 2015; 16:814.doi: 10.1186/s12864-015-2012-4 [PubMed: 26481321]

34. van der Veeken J, et al. Memory of Inflammation in Regulatory T Cells. Cell. 2016; 166:977-990. DOI: 10.1016/j.cell.2016.07.006 [PubMed: 27499023]

35. Dupuy AJ, Akagi K, Largaespada DA, Copeland NG, Jenkins NA. Mammalian mutagenesis using a highly mobile somatic Sleeping Beauty transposon system. Nature. 2005; 436:221-226. DOI: 10.1038/nature03691 [PubMed: 16015321]

36. Boeva V, et al. Control-FREEC: a tool for assessing copy number and allelic content using nextgeneration sequencing data. Bioinformatics. 2012; 28:423-425. DOI: 10.1093/bioinformatics/ btr670 [PubMed: 22155870] 
37. Hanzelmann S, Castelo R, Guinney J. GSVA: gene set variation analysis for microarray and RNAseq data. BMC Bioinformatics. 2013; 14:7.doi: 10.1186/1471-2105-14-7 [PubMed: 23323831]

38. Choi JK, et al. Hybrid HIV/MSCV LTR enhances transgene expression of lentiviral vectors in human CD34(+) hematopoietic cells. Stem cells. 2001; 19:236-246. DOI: 10.1634/stemcells. 19-3-236 [PubMed: 11359949]

39. Gazdar AF, Carney DN, Russell EK, Schechter GP, Bunn PA Jr. In vitro growth of cutaneous T-cell lymphomas. Cancer treatment reports. 1979; 63:587-590. [PubMed: 221120]

40. Morse HC 3rd, et al. Bethesda proposals for classification of lymphoid neoplasms in mice. Blood. 2002; 100:246-258. [PubMed: 12070034]

41. Rehg JE, Bush D, Ward JM. The utility of immunohistochemistry for the identification of hematopoietic and lymphoid cells in normal tissues and interpretation of proliferative and inflammatory lesions of mice and rats. Toxicologic pathology. 2012; 40:345-374. DOI: 10.1177/0192623311430695 [PubMed: 22434870] 

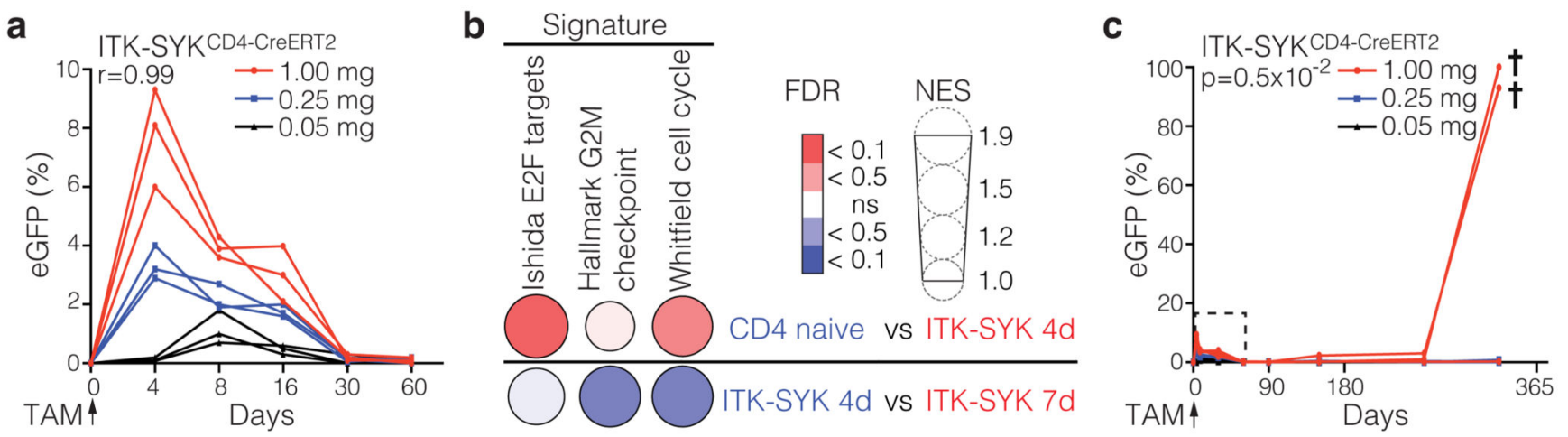

Figure 1. Counter-regulation of oncogenic $\mathbf{T}$ cell signalling in vivo.

a, Frequencies of ITK-SYK-expressing eGFP ${ }^{+}$lymphocytes in the blood of the ITK$\mathrm{SYK}^{\mathrm{CD} 4-C r e E R T 2}$ mice following single injections of tamoxifen ( $\mathrm{n}=3$ mice per dose). $\mathrm{r}=$ Pearson's correlation coefficient

b, Gene set enrichment analyses of wild-type naïve CD4 ${ }^{+} \mathrm{T}$ cells (CD4 naïve) and ITKSYK $^{\text {CD4-CreERT2 }}$ T cells at 4 (ITK-SYK 4d) and 7 days (ITK-SYK 7d) after tamoxifen injection. FDR=colour intensity of the circle. $\mathrm{NES}=$ circle diameter. Blue/red indicates the group in which a signature was positively enriched; $n=3$ biological replicates for each group. NES, normalised enrichment score; FDR, false discovery rate

c, Cohorts shown in a were followed over one year ( $n=3$ biological replicates per dose). The data in the dashed box correspond to the data in $\mathbf{a}$. $\dagger$ indicates animals that had to be euthanized because of lymphoma. $\mathrm{P}=$ Fisher's exact test. The test was performed on pooled data from two independent experiments. Representative data from one experiment are shown. 


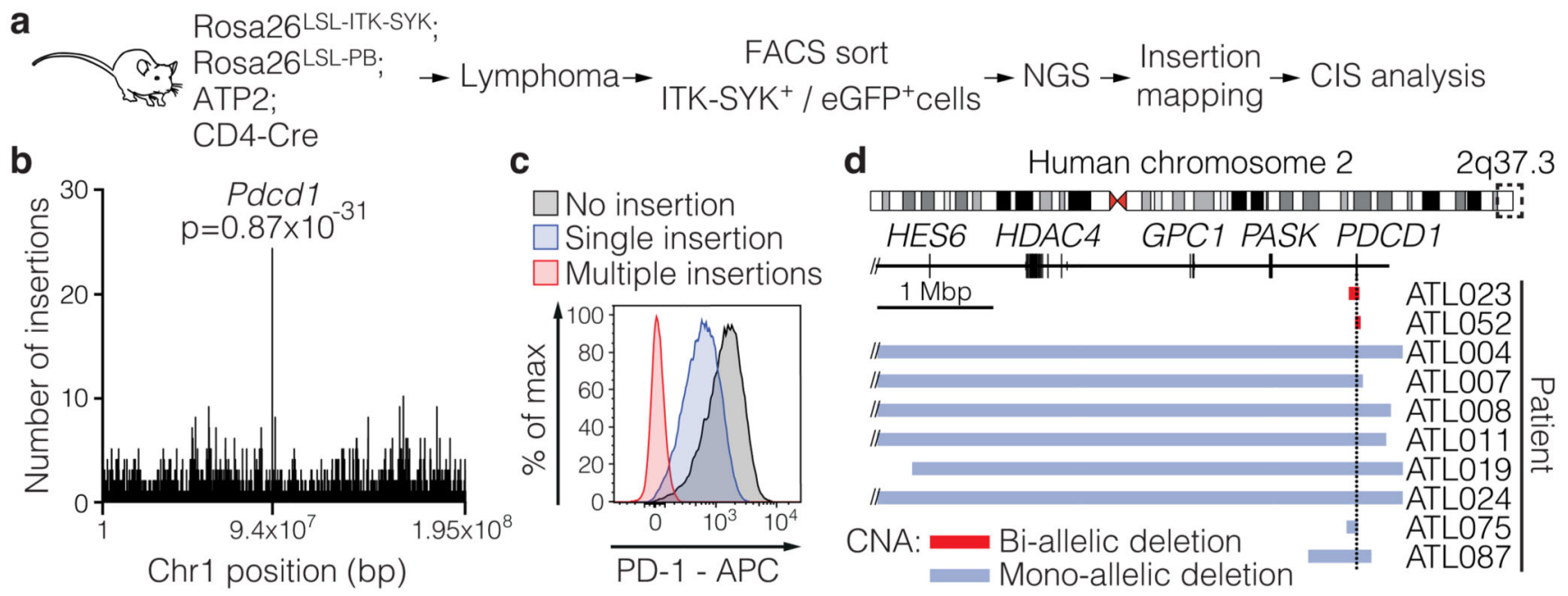

Figure 2. Identification of $P D C D 1$ alterations in $\mathrm{T}$ cell lymphoma.

a, Schematic representation of the transposon screen to discover T cell lymphoma genes.

Transposons from the ATP2 transgene were mobilized by piggyBac in ITK-SYK-expressing T cells. The tumour cells were then FACS-sorted based on eGFP expression, and the transposon insertion sites were identified by next-generation sequencing and bioinformatics analysis.

b, Transposon insertion densities within pooled lymphomas ( $\mathrm{n}=30$ mice) for the murine chromosome 1, total: 2732 insertions and Pdcd1 locus: 23 insertions. The p-value was calculated with a one-sided Poisson distribution based test, which assumes transposon insertions occurring at a constant rate.

c, Flow cytometric analysis of PD-1 expression on lymphoma cells from diseased

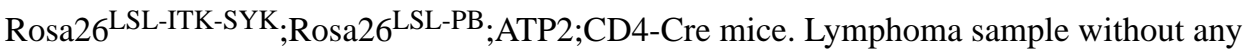
transposon cassette within the $P d c d 1$ gene (No insertion), with a single transposon cassette located in the Pdcd1 locus (Single insertion) or with multiple transposon cassettes within Pdcd1 (Multiple insertions).

d, The dashed box indicates the genomic region q37.3 on human chromosome 2 that is shown in detail in the lower part of the panel (top). Mono- or bi-allelic deletions of the PDCD1 gene detected in human T cell lymphoma patients are shown (bottom). The horizontal bars indicate the regions that were affected by the CNAs. The colour of the bar indicates the type of CNA. CNA, copy number aberration; Mbp, million base pairs c, Representative results from at least 15 mice. d, Results of an analysis that included all available WGS data within one published dataset. 
a

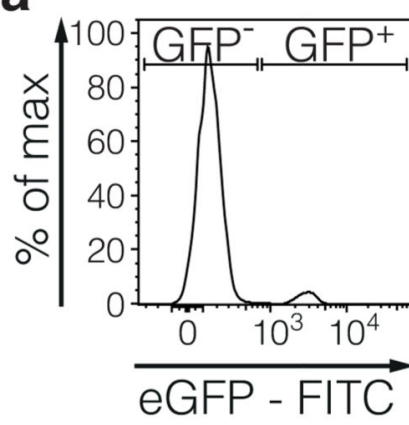

C

T-NHL patients $(n=50)$

Geneset:

GO antigen receptor mediated signaling pathway

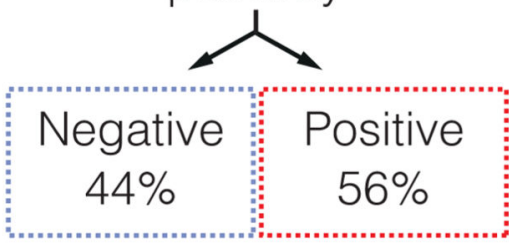

Enrichment score
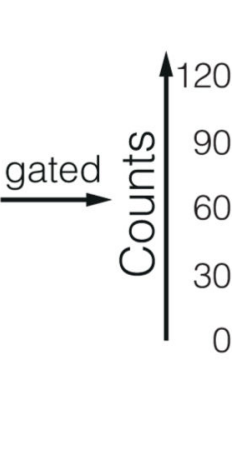

d
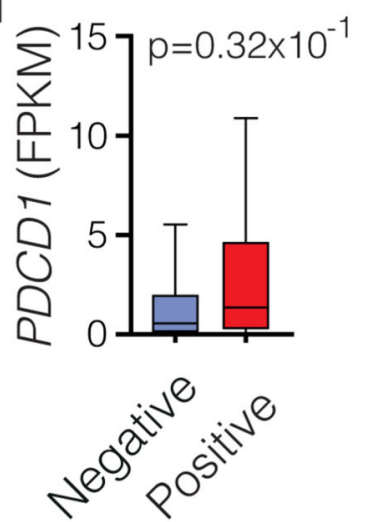

Enrichment score
$\mathrm{GFP}^{+}$

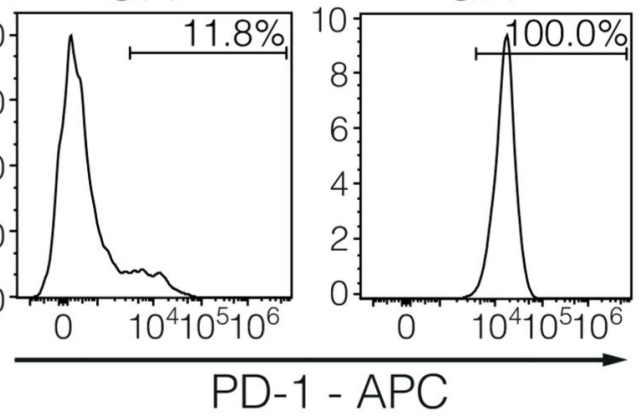

e

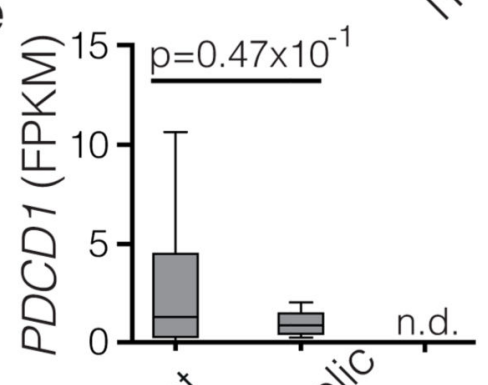

f $\square$ ITK-SYKCD4-CreERT2

口ITK-SYKCD4-CreERT2 + PD-L1
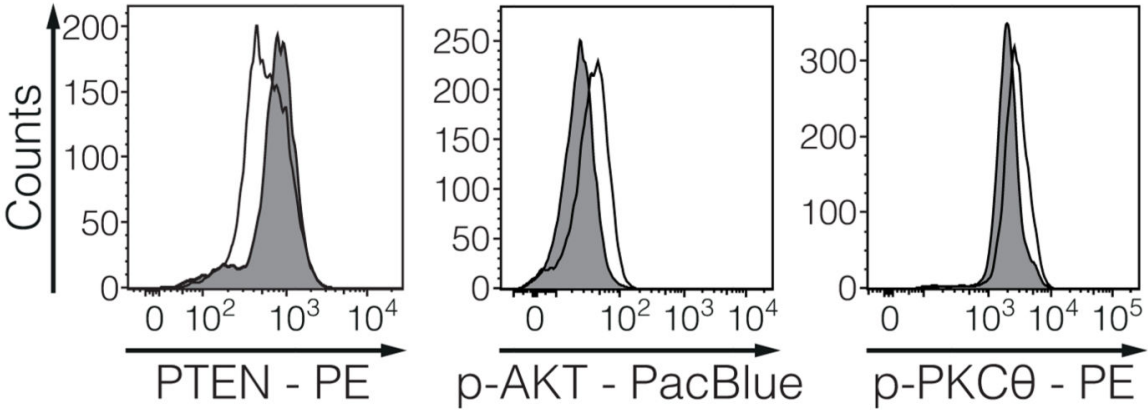

Figure 3. Oncogenic T cell signalling induces a PD-1 inhibitory loop.

a, PD-1 expression on the peripheral blood lymphocytes from an ITK-SYK ${ }^{\mathrm{CD} 4-C r e E R T 2}$ mouse as measured by flow cytometry 96 hours after tamoxifen injection.

b, Normalized transcript read counts of reads that were mapped to the Pdcd1 locus and detected in the RNAseq dataset from naïve $\mathrm{CD} 4^{+} \mathrm{T}$ cells (Naive) or ITK-SYK-expressing $\mathrm{eGFP}^{+} \mathrm{CD} 4^{+} \mathrm{T}$ cells from ITK-SYK ${ }^{\mathrm{CD}} 4-\mathrm{CreERT} 2$ mice 4 days after the tamoxifen injection (ITK-SYK). Shown are the mean \pm s.e.m and individual data points. FPKM, fragments per kilobase of exon per million fragments mapped; n.d., not detectable indicates FPKM $<0.1$; $\mathrm{n}=3$ mice per group. 
c, Gene Set Variation Analysis (GSVA) of fifty primary human T-NHL RNAseq samples is shown. The analysis was performed based on the geneset GO_antigen_receptor_mediated_signaling_pathway.

d, PDCD1 FPKM distributions from the samples in a are shown. The T-NHL patients $(\mathrm{n}=50)$ were classified according to their enrichment score. $\mathrm{P}=$ one-sided unequal variances Student's t-test. The FPKM distributions are shown as box plots. The 0.25-, 0.5- and 0.75quantile is depicted by the box, minimum and maximum by the whiskers.

e, $P D C D 1$ FPKM distributions from the positive samples in $\mathbf{d}$ are shown. The lymphomas were classified according to their PDCD1 copy number status. All T-NHLs with positive enrichment score were included $(\mathrm{n}=28)$. $\mathrm{P}=$ one-sided unequal variances Student's t-test. The FPKM distributions are shown as box plots. The 0.25-, 0.5- and 0.75-quantile is depicted by the box, minimum and maximum by the whiskers; n.d., not detectable indicates FPKM $<0.1$. f, Intracellular flow cytometric analyses of PTEN, p-AKT and p-PKC $\theta$ levels in primary ITK-SYK-expressing T cells stimulated with PD-L1 or Fc-fragment-coated microspheres. a, Representative results from three analysed mice. f, Representative data from three independent experiments with two biological replicates per experiment. 

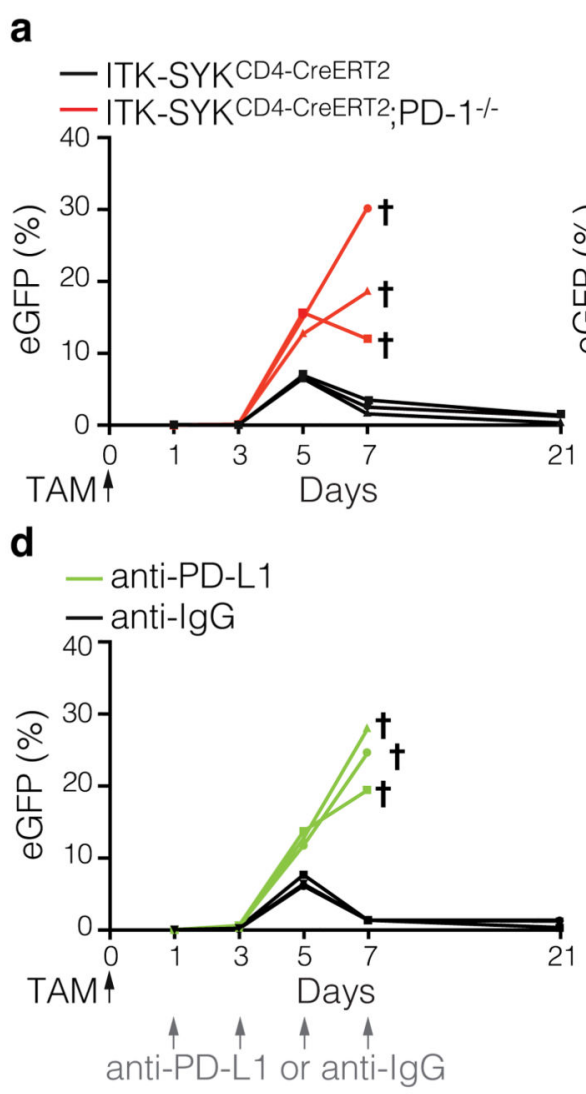

g $\square$ Rosa26LSL-ITK-SYK TAT-CRE treated

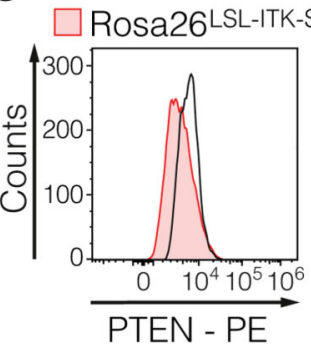

b

e
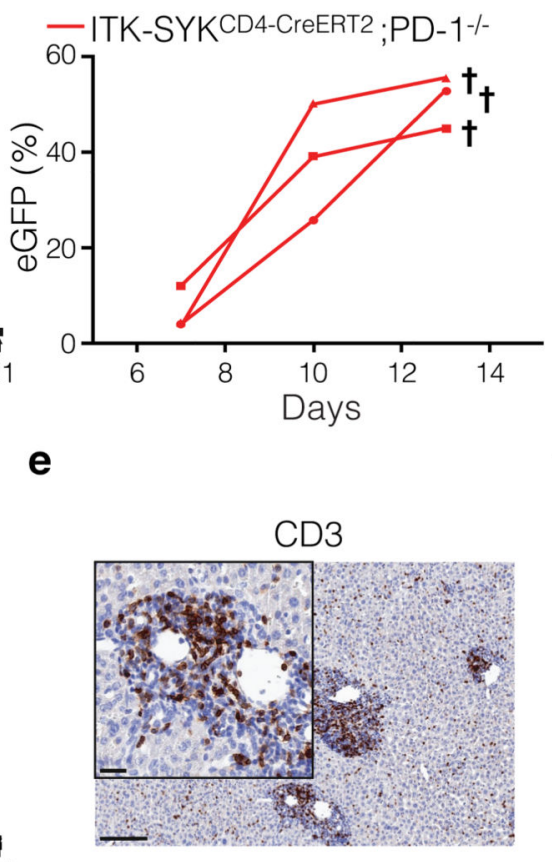

$$
\text { f }
$$

f

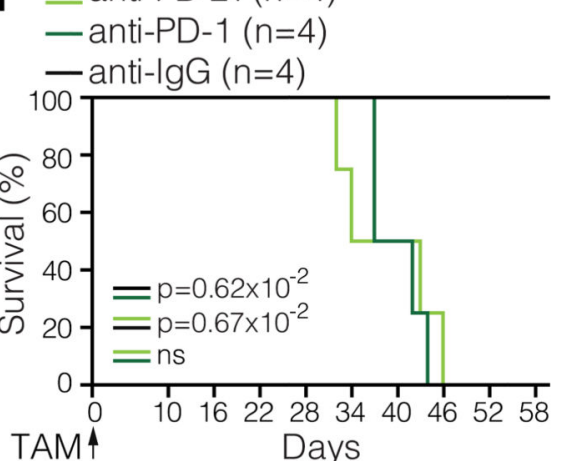

TAM 4

$A \uparrow \uparrow \uparrow \uparrow \uparrow \uparrow \uparrow \uparrow \uparrow \uparrow \uparrow \uparrow \uparrow \uparrow \uparrow \uparrow$ anti-PD-L1, anti-PD-1 or anti-IgG

h

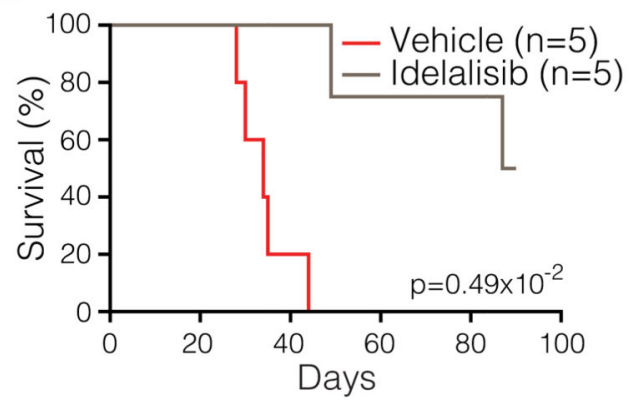

Figure 4. PD-1 is a haploinsufficient tumour suppressor in vivo. a, ITK-SYK ${ }^{\text {CD4-CreERT2 }}$ and ITK-SYK ${ }^{\text {CD4-CreERT2 }}$;PD- $1^{-/-}$mice ( $\mathrm{n}=3$ mice per genotype) received single tamoxifen injections $(0.25 \mathrm{mg})$. The percentages of ITK-SYK-expressing $\mathrm{eGFP}^{+}$blood lymphocytes were determined using flow cytometry.

b, Fifty million splenic cells from diseased ITK-SYK ${ }^{\mathrm{CD} 4-\mathrm{CreERT} 2} ; \mathrm{PD}-1^{-/-}$mice $(\mathrm{n}=3$ donors $)$ were intravenously transferred to NSG mice ( $\mathrm{n}=3$ recipients). The percentage of eGFP ${ }^{+}$ lymphocytes in the recipient's blood is indicated. NSG, NOD SCID II $\mathrm{rg}^{-/-}$ c, Survival of ITK-SYK ${ }^{\mathrm{CD} 4-C r e E R T 2}$, ITK-SYK ${ }^{\mathrm{CD} 4-\mathrm{CreERT}}$;PD-1 ${ }^{+/-}$and ITK$\mathrm{SYK}^{\mathrm{CD} 4-\mathrm{CreERT}}$;PD- ${ }^{-/-}$mice $(\mathrm{n}=6, \mathrm{n}=12$ and $\mathrm{n}=14)$. All mice received a single tamoxifen dose on day zero. $\mathrm{P}=$ two-sided log-rank test d, The percentages of ITK-SYK-expressing eGFP ${ }^{+}$peripheral blood lymphocytes of

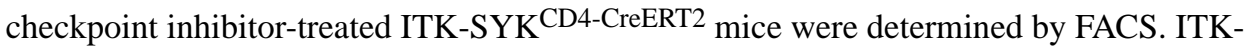


$\mathrm{SYK}^{\mathrm{CD} 4-C r e E R T 2}$ mice received single tamoxifen injections. Beginning twenty-four hours later, the mice received $200 \mu \mathrm{g}$ of anti-PD-L1 or control antibody ( $\mathrm{n}=3$ mice per condition) every second day.

e, Anti-CD3 antibody-stained liver section from a diseased anti-PD-L1-treated ITK$\mathrm{SYK}^{\mathrm{CD} 4-\mathrm{CreERT} 2}$ mouse from the experiment shown in d, Scale bars represent $100 \mu \mathrm{m}$ and $20 \mu \mathrm{m}$ (inset).

f, Survival of ITK-SYK ${ }^{\text {CD4-CreERT2 }}$ mice that received checkpoint inhibitor treatment

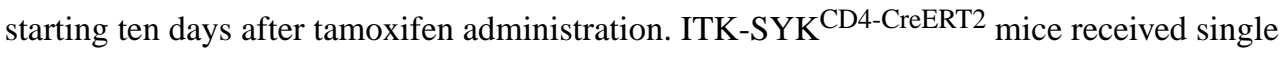
injections of tamoxifen on day zero $(0.25 \mathrm{mg})$. Starting ten days after injection, mice were administered $200 \mu \mathrm{g}$ of anti-PD-L1 or anti-PD-1 antibody or control antibody ( $\mathrm{n}=4$ mice per condition) every third day. $\mathrm{P}=$ two-sided log-rank test

g, Phosflow analyses of PTEN, p-AKT and p-PKC $\theta$ levels in ITK-SYK-expressing T cells isolated from the C57BL/6 recipients presented in Extended Data Figure 6b. The genotype of the transplanted cells is indicated.

h, Survival of the PI3K $\delta$ inhibitor- or vehicle-treated recipient mice. Tamoxifen-induced ITK-SYK ${ }^{\mathrm{CD} 4-C r e E R T 2}$;PD- ${ }^{-/-}$T cells ( $\mathrm{n}=5$ biological replicates, $5 \times 10^{4}$ cells per transplant) were transplanted into NSG mice ( $\mathrm{n}=5$ recipients per group). $\mathrm{P}=$ two-sided log-rank test. $\dagger$ indicates animals that were euthanized because of lymphomas. a, Representative data from three independent experiments, each with three biological replicates. $\mathbf{b}$, The data from a single experiment that was independently repeated once with comparable results. c, Pooled data from two independent experiments. $\mathbf{d}$, Representative data from four independent experiments, each with three biological replicates per antibody. e, Representative histology from one out of nine analysed mice. f, Data from a single experiment, which was repeated once with similar results. g, Representative results from two out of eight analysed mice in two independent experiments. $\mathbf{h}$, Pooled survival data from two experiments. 